\title{
Identification of dynamic models in complex networks with prediction error methods : basic methods for consistent module estimates
}

Citation for published version (APA):

Hof, Van den, P. M. J., Dankers, A. G., Heuberger, P. S. C., \& Bombois, X. (2013). Identification of dynamic models in complex networks with prediction error methods: basic methods for consistent module estimates. Automatica, 49(10), 2994-3006. https://doi.org/10.1016/j.automatica.2013.07.011

DOI:

10.1016/j.automatica.2013.07.011

Document status and date:

Published: 01/01/2013

Document Version:

Accepted manuscript including changes made at the peer-review stage

Please check the document version of this publication:

- A submitted manuscript is the version of the article upon submission and before peer-review. There can be important differences between the submitted version and the official published version of record. People interested in the research are advised to contact the author for the final version of the publication, or visit the DOI to the publisher's website.

- The final author version and the galley proof are versions of the publication after peer review.

- The final published version features the final layout of the paper including the volume, issue and page numbers.

Link to publication

\footnotetext{
General rights

- You may freely distribute the URL identifying the publication in the public portal. follow below link for the End User Agreement:

www.tue.nl/taverne

Take down policy

If you believe that this document breaches copyright please contact us at:

openaccess@tue.nl

providing details and we will investigate your claim.
}

Copyright and moral rights for the publications made accessible in the public portal are retained by the authors and/or other copyright owners and it is a condition of accessing publications that users recognise and abide by the legal requirements associated with these rights.

- Users may download and print one copy of any publication from the public portal for the purpose of private study or research.

- You may not further distribute the material or use it for any profit-making activity or commercial gain

If the publication is distributed under the terms of Article 25fa of the Dutch Copyright Act, indicated by the "Taverne" license above, please 


\title{
Identification of Dynamic Models in Complex Networks with Prediction Error Methods - Basic Methods for Consistent Module Estimates *
}

\author{
Paul M. J. Van den Hof ${ }^{a}$, Arne Dankers ${ }^{b}$, Peter S.C. Heuberger ${ }^{c}$ and Xavier Bombois ${ }^{b}$ \\ ${ }^{a}$ Department of Electrical Engineering, Eindhoven University of Technology, The Netherlands \\ ${ }^{\mathrm{b}}$ Delft Center for Systems and Control, Delft University of Technology, The Netherlands \\ ${ }^{\mathrm{c}}$ Department of Mechanical Engineering, Eindhoven University of Technology, The Netherlands
}

\begin{abstract}
The problem of identifying dynamical models on the basis of measurement data is usually considered in a classical open-loop or closedloop setting. In this paper this problem is generalized to dynamical systems that operate in a complex interconnection structure and the objective is to consistently identify the dynamics of a particular module in the network. For a known interconnection structure it is shown that classical prediction error methods for closed-loop identification can be generalized to provide consistent model estimates, under specified experimental circumstances. Two classes of methods considered in this paper are the direct method and the joint-IO method that rely on consistent noise models, and indirect methods that rely on external excitation signals like two-stage and IV methods. Graph theoretical tools are presented to verify the topological conditions under which the several methods lead to consistent module estimates.
\end{abstract}

Key words: System identification; closed-loop identification; graph theory; dynamic networks; identifiability; linear systems.

\section{Introduction}

One of the challenges in the systems and control field is to develop effective synthesis methods for distributed control of systems that operate in a network structure. While considerable attention is devoted to this problem from a model-based control perspective, attention for the underlying modelling problem is much more limited. In particular the problem of identifying dynamical models on the basis of measurement data that is obtained from a (complex) dynamic network, and where use can be made of external probing/excitation signals, has not been addressed in much detail yet. At the same time, modelling of interconnected systems is playing an increasingly important role in many fields of science and

\footnotetext{
* Originally submittted to Automatica, 26 October 2012. Revised 2 April 2013. Final version 21 June 2013. This paper was not presented at any IFAC meeting. Corresponding author: Paul M.J. Van den Hof. The work of Arne Dankers is supported in part by the National Science and Engineering Research Council (NSERC) of Canada.

Email addresses: p.m.j.vandenhofetue.nl (Paul M. J. Van den Hof), a.g.dankers@tudelft.nl (Arne Dankers), p.s.c.heubergeretue.nl (Peter S.C. Heuberger), x.j.a.bombois@tudelft.nl (Xavier Bombois).
}

engineering: power systems, biological systems, flexible mechanical structures, economic systems, to name a few.

In this paper we will consider identification problems in networks of dynamic systems. From an identification perspective this can be considered as a natural extension of the situation of open-loop data, closed-loop data in a single loop, towards data that is obtained from systems operating in a predefined network structure, where some of the modules may have known dynamics (as e.g. a particular controller in the network). Since dynamic networks typically contain (feedback) loops, it is expected that methods for closed-loop identification are an appropriate basis for developing more generalized tools to deal with complex networks.

In our framework discussed here, a dynamic network is defined as an interconnection of transfer functions or modules where the interconnecting signals (terminals) are considered as nodes/vertices in the network, and proper transfer functions are considered as links/edges. In this paper it will be assumed that the interconnection structure (topology) of the network is known, and our goal is to identify the dynamics of a single module or a collection of modules in the network.

Classical methods for closed-loop identification are ad- 
dressed in $[9,20]$. For a single contribution to the problem of structured systems see also [14]. Much of the dynamic network identification literature focuses on identifying the dynamics of the full network, or on detecting the topology of the network. Nonparametric methods have been used to detect the structure of dynamic networks in $[17,16,19,11]$ (without worrying about obtaining consistent estimates of the dynamics of the network). Consistency-based parametric identification of the interconnection structure is addressed in $[3,25,26,5]$. The conditions under which the network identification algorithms work typically include the condition that all disturbance/noise processes are uncorrelated and should be modelled exactly in conjunction with the dynamic transfer functions in the network. However in a large-scale dynamic network it can be questioned if this is realistic. In this paper we therefore will particularly also include identification methods that can consistently identify dynamic transfer functions without relying on exact noise models, and where weaker assumptions on the noise sources are considered.

Taking classical principles of closed-loop identification as a starting point we will analyze the sketched problem by generalizing several closed-loop identification methods to the dynamic network situation. The direct method [15] will typically rely on exact noise models (system in the model set). This also similarly for the joint-IO method. On the other hand, the closed-loop identification methods referred to as two-stage method [22] and instrumental variable method [13] typically rely on the presence of external excitation or probing signals. They also will be generalized to deal with the dynamic network situation. Throughout the paper we will focus on conditions on the interconnection structure as well as on the presence of noise sources and excitation/probing signals for consistently identifying a particular module in the network.

The paper will proceed as follows. In Section 2 a particular setup of dynamic network is chosen and defined, and the problem under investigation will be formally stated. In Section 3 some background information will be presented on Prediction-Error identification, the Direct and Two Stage closed loop methods, and some graph theoretical results. Next some network properties are presented in Section 4. In Sections 5 and 6 the Direct and Two-Stage Methods for interconnected systems will be presented and analyzed. Finally some concluding remarks will be made.

Notation: $[\cdot]_{j i}$ is matrix element $(j, i)$ of the matrix $[\cdot]$.

The research presented in this paper further builds on the preliminary results presented in $[4,23]$.

\section{System setup}

\subsection{Dynamic network and problem setup}

The network structure that we consider in this paper is built up of $L$ elements or nodes, related to $L$ measured scalar signals $w_{j}, j=1, \cdots L$. Every node signal $w_{j}$ in this network is written as:

$$
w_{j}(t)=\sum_{k \in \mathcal{N}_{j}} G_{j k}^{0}(q) w_{k}(t)+r_{j}(t)+v_{j}(t)
$$

with $G_{j k}^{0}(z)$ a proper rational transfer function,

- $\mathcal{N}_{j}$ the set of indices of node signals $w_{k}, k \neq j$, for which $G_{j k}^{0} \neq 0$, i.e. the set of indices of measured variables with direct causal connections to $w_{j}$;

- $v_{j}$ an unmeasured disturbance signal being a realization of a stationary stochastic process with rational spectral density, represented by $v_{j}=H_{j}^{0}(q) e_{j}$ with $e_{j}$ a white noise process and $H_{j}^{0}$ a monic, stable and stably invertible filter;

- $r_{j}$ an external excitation signal, quasi-stationary [15], that is available to (and possibly can be manipulated by) the user,

- $q^{-1}$ the delay operator $q^{-1} u(t)=u(t-1)$, and

- signals $v_{j}$ and $r_{j}$ may or may not be present.

All the measured variables can be written in a single matrix equation as:

$$
\begin{aligned}
{\left[\begin{array}{c}
w_{1} \\
w_{2} \\
\vdots \\
w_{L}
\end{array}\right] } & =\left[\begin{array}{cccc}
0 & G_{12}^{0} & \cdots & G_{1 L}^{0} \\
G_{21}^{0} & 0 & \ddots & G_{2 L}^{0} \\
\vdots & \ddots & \ddots & \vdots \\
G_{L 1}^{0} & G_{L 2}^{0} & \cdots & 0
\end{array}\right]\left[\begin{array}{c}
w_{1} \\
w_{2} \\
\vdots \\
w_{L}
\end{array}\right]+\left[\begin{array}{c}
r_{1} \\
r_{2} \\
\vdots \\
r_{L}
\end{array}\right]+\left[\begin{array}{c}
v_{1} \\
v_{2} \\
\vdots \\
v_{L}
\end{array}\right] \\
& =G^{0} w+r+v \\
& =\left(I-G^{0}\right)^{-1}(r+v)
\end{aligned}
$$

where in the latter equation it is assumed that the inverse $\left(1-G^{0}\right)^{-1}$ exists.

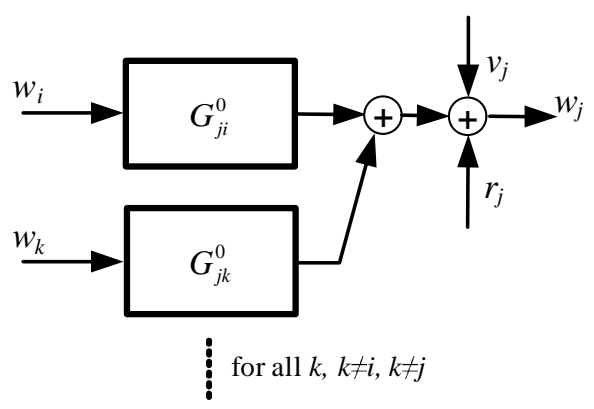

Fig. 1. Single building block in a network structure related to the construction of node signal $w_{j}$.

A single building block of the network related to the construction of node signal $w_{j}$ is sketched in Figure 1, where the transfer function $G_{j i}^{0}$ has been separately indicated to focus on the module that is supposed to be identified.

A dynamic network is then constructed by interconnecting the several blocks through their node signal. For a particular 


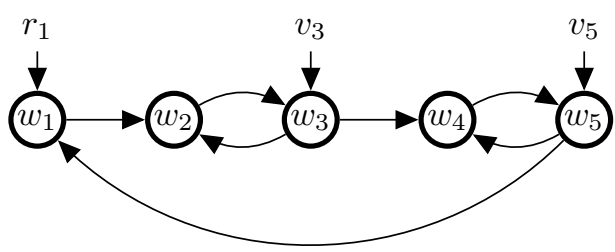

(a).

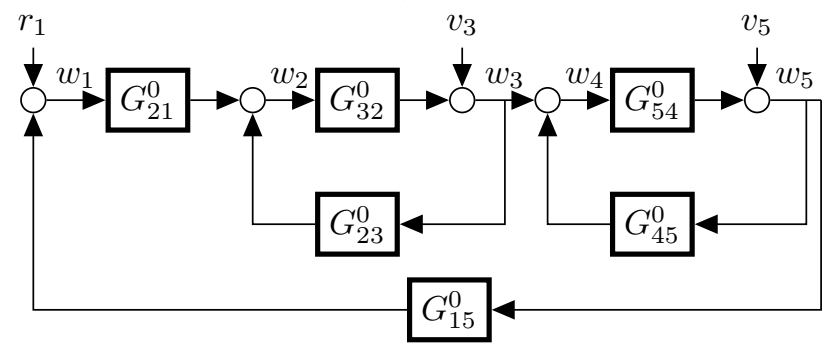

(b).

Fig. 2. Two popular visualizations of the same dynamic network. (a) Node-and-Link visualization (measurement-centric). Each node represents a measured variable. The links between node $w_{i}$ and node $w_{j}$ represents a transfer function $G_{j i}^{0}$. (b) Transfer function representation (module-centric). Each block represents a transfer function between two measured variables $w_{i}$ and $w_{j}$. The signals are added together by adders represented as circles.

example this is visualized in Figure 2. It shows a node-andlink visualization (Fig. 2a) that is popular in many fields such as artificial intelligence, machine learning [17], biological systems [26], where the arrows between signals $w_{j}$ represent causal relationships. It emphasizes the links between measured variables (measurement-centric). The second visualization is a transfer function based visualization (Figure 2b) (module-centric), commonly used in control.

All node signals $w_{j}, j=1, \cdots L$ are supposed to be measurable, while at each node a noise signal $v_{j}$ (non-measurable) and excitation signal $r_{j}$ (measurable) may or may not be present. Each excitation signal $r_{j}$ is assumed to be uncorrelated to all noise signals $v_{i}$. Some parts of the network may have dynamics that are known a priori. This is e.g. the case in a classical closed-loop system with a known controller. Since all node signals are supposed to measured, and the network structure is supposed to be known, we do not face the problem of ambiguous or hidden states/variables, see e.g. [24].

The problem that will be addressed in this paper is: given the interconnection structure of the dynamic network, specify conditions under which a particular module transfer function $G_{j i}^{0}$ can be estimated consistently.

The conditions for consistency of an identified module, will typically involve the interconnection structure of the network, and the presence and properties of disturbance and excitation signals.

One might think that identifying the full dynamic network can always be cast into a -standard- multivariable closedloop identification problem, while relying on classical meth- ods to resolve this. However the problem of deciding which variables to classify as inputs and outputs, or to include structural constraints (zero modules, known modules), and to determine the "minimum" excitation conditions under which consistent identification is possible, makes this nontrivial. By focussing on one particular module $G_{j i}^{0}$ we intend to reveal these structural phenomena in the identification problem.

Different approaches will be presented and analyzed that can address the formulated problem. They are direct generalizations of classical closed-loop identification methods:

- Direct identification [15], with an emphasis on including the identification of exact noise/disturbance models;

- Indirect identification, such as the two-stage method and the IV-method [22,13], with an emphasis on the use of external excitation or probing signals.

- Joint-IO identification [1], where input and output signals are modelled as the output of a stationary stochastic process.

In general each of the several methods will be able to identify the module $G_{j i}^{0}$ in the network, dependent on the presence of noise and excitation signals, the network topology and the presence of a priori known transfers. This will be analyzed in the rest of this paper.

The dynamic networks that we consider are assumed to satisfy the following general conditions.

Assumption 1 We consider a dynamic network of which one building block, leading to the construction of node signal $w_{j}$, is depicted in Figure 1, with the additional properties that

a. All module transfer functions in the network are proper;

$b$. The network is well-posed in the sense that all minors of $\left(I-G^{0}(\infty)\right)$ are non-zero ${ }^{1}$;

c. $\left(I-G^{0}\right)^{-1}$ is proper and stable.

$d$. The vector noise process $v:=\left(v_{1}, \cdots v_{L}\right)^{T}$ has a positive semi-definite spectral density, $\Phi_{v}(\omega) \geq 0$, not necessarily diagonal;

\subsection{Network topology and graph theory}

We will need some further notation to characterize the network interconnection (topology), as well as some tools from graph theory.

For the specification of the topology of the network we will utilize a directed graph that indicates the locations and causal directions of module transfers in the network. It can be represented by an adjacency matrix $A \in \mathbb{R}^{L \times L}$, defined as:

$$
\begin{array}{ll}
A(j, i)=0 & \text { if } G_{j i}^{0}(q) \equiv 0 \\
A(j, i)=1 & \text { elsewhere. }
\end{array}
$$

\footnotetext{
1 The property of well-posedness is adopted from [2]. It imposes (weak) restrictions on allowable feed-through terms in the network but still allows the occurrence of algebraic loops.
} 
Because of the interconnection structure that we consider here (see (1)) it follows that $A(i, i)=0, i=1, \cdots L$.

The following lemma from graph theory will be very useful :

Lemma 1 [8] Consider a directed graph with adjacency matrix $A$. Then for $k \geq 1,\left[A^{k}\right]_{j i}$ indicates the number of different path connections of length $k$ from node $i$ to node $j$.

In addition to the adjacency matrix $A$ defined above, we will also consider a related delay-adjacency matrix $A_{d}$ of which the elements have three possible values: 0 (no link), 1 (a link with no delay), and $d$ (a link with a delay). Through the use of the following rules for addition and multiplication:

$$
\begin{array}{llll}
0+0=0 & 0+d=d & 0 \cdot 0=0 & 0 \cdot d=0 \\
0+1=1 & 1+d=1 & 0 \cdot 1=0 & 1 \cdot d=d \\
1+1=1 & d+d=d & 1 \cdot 1=1 & d \cdot d=d
\end{array}
$$

summation and multiplication of matrices $A_{d}$ can be defined, and one can evaluate $\left[A_{d}^{k}\right]_{j i}$. The following lemma will be helpful in characterizing the presence of delays in particular network loops. See also e.g. [8].

Lemma 2 Consider a directed graph with delay-adjacency matrix $A_{d}$ and the rules of multiplication and addition with d. Then for $k \geq 1$,

- $\left[A_{d}^{k}\right]_{j i}=1$ indicates that there is a path of length $k$ from $i$ to $j$ without a delay,

- $\left[A_{d}^{k}\right]_{j i}=d$ indicates that all paths of length $k$ from $i$ to $j$ have a delay,

- $\left[A_{d}^{k}\right]_{j i}=0$ indicates that there is no path of length $k$ from $i$ to $j$.

We will further consider the following sets:

- $\mathcal{V}$ denotes the set of indices of node signals to which additive noise sources $v$ are directly connected.

- $\mathcal{R}$ denotes the set of indices of node signals to which external excitation signals $r$ are directly connected.

- $\mathcal{V}_{j}$ is the subset of $\mathcal{V}$ of indices of noise signals for which there exists a path to $w_{j}$;

- $\mathcal{R}_{j}$ is the subset of $\mathcal{R}$ of indices of excitation signals for which there exists a path to $w_{j}$;

- $\mathcal{K}_{j}$ denotes the set of indices of node signals $w_{k}, k \in \mathcal{N}_{j}$ for which the module transfers $G_{j k}$ are known;

- $\mathcal{U}_{j}^{i}$ denotes the set of indices of node signals $w_{k}, k \in$ $\mathcal{N}_{j}, k \neq i$ for which the module transfers $G_{j k}$ are unknown.

Note that if $i \in \mathcal{N}_{j}$ then $\mathcal{N}_{j}=i \cup \mathcal{K}_{j} \cup \mathcal{U}_{j}^{i}$. In the sequel variables $j$ and $i$ are used to denote the particular module $G_{j i}^{0}$ that we intend to identify.

\section{Prediction error identification and extension to dy-} namic networks

\subsection{Prediction error identification}

For reviewing a standard prediction error identification method we express a measured variable $w_{j}$ which is chosen as output variable, as a function of a particular set of variables $\left\{x_{1}, x_{2}, \ldots,\right\}=\mathcal{X}$, which are chosen as the inputs. Inputs can be external excitations $r_{k}$ or other measured variables $w_{k}, k \neq j$. We assume that the output can be expressed in terms of the inputs as

$$
w_{j}(t)=\sum_{x_{k} \in \mathcal{X}} G_{j k}^{0}(q) x_{k}(t)+H_{j}^{0}(q) \tilde{e}_{j}(t)+r_{j}(t),
$$

with $G_{j i}^{0}$ proper transfer functions, $H_{j}^{0}$ a stable and stably invertible transfer, and $\tilde{e}_{j}$ a white noise process. Note that in the situation of the previous sections, this assumption holds e.g. if we choose $x_{k}=w_{k}, \mathcal{X}=\mathcal{N}_{j}$ and $\tilde{e}_{j}=e_{j}$.

The module transfers $G_{j k}^{0}$ and noise filter $H_{j}^{0}$ are modelled using parametrized transfer functions $G_{j k}(q, \theta)$ and $H_{j}(q, \theta)$, and the one-step-ahead predictor for $w_{j}$ is given by $([15])$ :

$$
\begin{aligned}
\hat{w}_{j}(t \mid t-1 ; \theta)- & r_{j}(t)=\left(1-H_{j}^{-1}(q, \theta)\right)\left(w_{j}(t)-r_{j}(t)\right)+ \\
& +H_{j}^{-1}(q, \theta)\left(\sum_{x_{k} \in \mathcal{X}} G_{j k}(q, \theta) x_{k}(t)\right),
\end{aligned}
$$

or equivalently

$$
\begin{aligned}
\hat{w}_{j}(t \mid t & -1 ; \theta)=\left(1-H_{j}^{-1}(q, \theta)\right) w_{j}(t)+ \\
& +H_{j}^{-1}(q, \theta)\left(\sum_{x_{k} \in \mathcal{X}} G_{j k}(q, \theta) x_{k}(t)+r_{j}(t)\right) .
\end{aligned}
$$

The unknown parameters are estimated through a quadratic prediction error criterion:

$$
\hat{\theta}_{N}=\arg \min _{\theta} V_{N}(\theta)
$$

with $V_{N}(\theta)=\frac{1}{N} \sum_{t=0}^{N-1} \varepsilon_{j}^{2}(t, \theta)$ and the prediction error $\varepsilon_{j}(t, \theta):=w_{j}(t)-\hat{w}_{j}(t \mid t-1 ; \theta)$.

Under standard -weak- assumptions the estimated parameter converges in the number of data $N$, to satisfy ([15]):

$$
\hat{\theta}_{N} \rightarrow \theta^{*} \text { w.p. } 1 \text { as } N \rightarrow \infty
$$

with $\theta^{*}=\arg \min _{\theta} \overline{\mathbb{E}}\left[\varepsilon_{j}^{2}(t, \theta)\right]$, with $\overline{\mathbb{E}}:=\lim _{N \rightarrow \infty} \frac{1}{N} \sum_{t=0}^{N-1} \mathbb{E}$, and $\mathbb{E}$ the expectation operator. If $G_{j k}\left(q, \theta^{*}\right)=G_{j k}^{0}(q)$ the module transfers are estimated consistently. Consistency is possible under several different conditions, dependent on the experimental circumstances, and the chosen model parametrizations. 


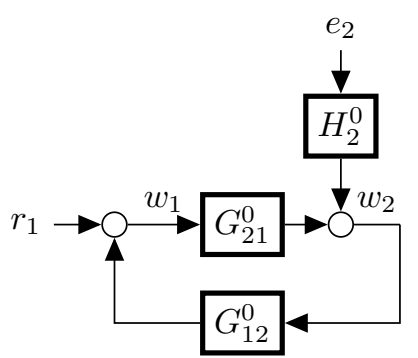

Fig. 3. Closed loop data generating system.

\subsection{Direct method of closed-loop identification}

In a simple closed-loop data generating system, as depicted in Figure 3, with the objective to identify the module transfer $G_{21}^{0}$, the direct method of closed-loop identification comes down to choosing $w_{2}$ as output, $w_{1}$ as input, and then applying the identification method as sketched in the previous section, with a parametrized predictor determined by $G_{21}(q, \theta)$ and $H_{2}(q, \theta)$.

Typical conditions for consistency of the estimate of the module transfer are

- $G_{21}^{0}$ and $H_{2}^{0}$ are present in the parametrized model set generated by $G_{21}(q, \theta)$ and $H_{2}(q, \theta)$ respectively. This is referred to as "system in the model set";

- the feedback loop is sufficiently excited with external signals $r_{1}$ and/or $e_{2}$;

- Plant feedback loop and model feedback loop should have at least one time-step delay (no algebraic loop).

A generalization of this method to the dynamic network case is obtained by applying the predictor model (4) for a particular output signal $w_{j}$ and selecting $x_{k}$ as a subset of the set of node signals $\left\{w_{k}\right\}_{k \in[1, L] \backslash j}$.

\subsection{Two-stage method of closed-loop identification}

The two-stage or projection method of closed-loop identification $([22,10])$ uses the same predictor structure $(4)$, to address the closed loop identification problem in Figure 3. However instead of using predictor input $x_{k}=w_{1}$, it uses the part of $w_{1}$ that is correlated to the external signal $r_{1}$. Note that $r_{1}$ and $w_{1}$ are quasi-stationary signals ([15]) such that the cross-correlation function

$$
R_{w_{1} r_{1}}(\tau):=\overline{\mathbb{E}}\left[w_{1}(t) r_{1}(t-\tau)\right]
$$

is zero for $\tau<0$ and non-zero for $\tau \geq 0$. Then there exists a proper transfer function $F_{w_{1} r_{1}}^{0}$ such that

$$
w_{1}(t)=F_{w_{1} r_{1}}^{0}(q) r_{1}(t)+z(t)
$$

with $z$ uncorrelated to $r_{1}$. This provides a decomposition

$$
w_{1}(t)=w_{1}^{\left(r_{1}\right)}(t)+w_{1}^{\left(\perp r_{1}\right)}(t)
$$

with $w_{1}^{\left(\perp r_{1}\right)}(t)=z(t)$. Note that $w_{1}^{\left(r_{1}\right)}$ is the projection of signal $w_{1}$ onto the space of (causally) time-shifted versions of $r_{1}$.

If $r_{1}$ and $w_{1}$ are available from measurements then $F_{w_{1} r_{1}}^{0}(q)$ can be consistently estimated from data, provided that the signal $r_{1}$ is persistently exciting of a sufficiently high order. This consistent estimation can be done without the necessity to model the noise dynamics of $z$, because it is essentially an open-loop type of identification problem $\left(r_{1}\right.$ and $z$ are uncorrelated). Subsequently the projection

$$
\hat{w}_{1}^{\left(r_{1}\right)}(t):=\hat{F}_{w_{1} r_{1}}(q) r_{1}(t)
$$

can be calculated, with $\hat{F}_{w_{1} r_{1}}(q)$ the estimated transfer. This estimate then can serve as an accurate estimate of $w^{(r)}(t)$. In the second step of the algorithm $\hat{w}_{1}^{\left(r_{1}\right)}$ is used as an input in the predictor model (4), determined by $G_{21}(q, \theta)$ and $\mathrm{H}_{2}(q, \theta)$.

Typical conditions for consistency of the estimate of the module transfer $G_{21}$ are

- In the first step a consistent estimate should be obtained of $F_{w_{1} r_{1}}$. This is typically achieved by high order modelling.

- If in the second stage $G_{21}(q, \theta)$ and $H_{2}(q, \theta)$ are parametrized independently, then only $G_{21}^{0}$ needs to be an element in the parametrized model set;

- the feedback loop is sufficiently excited with external signals $r_{1}$

- There are no conditions on (absence of) algebraic loops in the feedback system.

The generalization of this method to the dynamic network case can now be made as follows, and as illustrated in Figure 4.

(1) Find an external excitation signal $r_{m}$ that is correlated to $w_{i}$;

(2) Project $w_{i}$ onto $r_{m}$;

(3) Identify $G_{j i}^{0}$ by applying a predictor model $\hat{w}_{j}(t \mid t-$ $1, \theta)$ leading to a prediction error $\varepsilon_{j}(t, \theta)=$

$$
H_{j}(q, \eta)^{-1}\left[w_{j}(t)-G_{j i}(q, \theta) \hat{w}_{i}^{\left(r_{m}\right)}(t)-r_{j}(t)\right]
$$

where $H_{j}$ and $G_{j i}$ are parametrized independently, and a prediction error criterion such as (5).

\subsection{Joint-IO method of closed-loop identification}

The classical joint input-output method of closed loop identification, see e.g. [18,1], models the vector signal composed of $w_{1}$ and $w_{2}$ in Figure 3, as a stationary stochastic process that is driven by independent identically distributed random variables (white noise), according to

$$
\left(\begin{array}{l}
w_{2} \\
w_{1}
\end{array}\right)=\Upsilon_{0}(q)\left(\begin{array}{l}
e_{2} \\
e_{1}
\end{array}\right),
$$




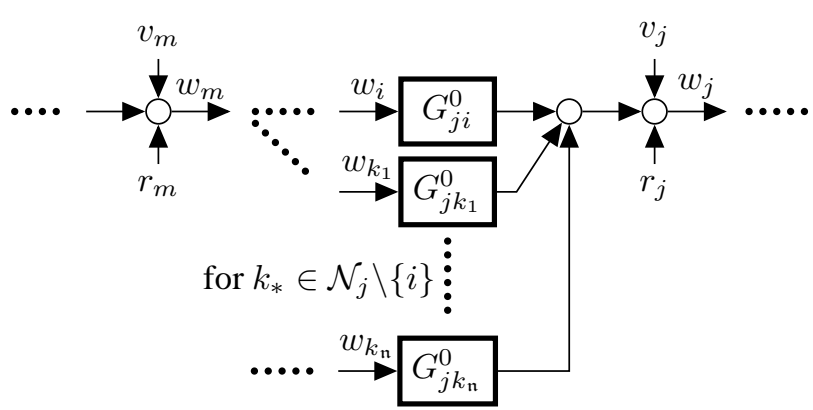

Fig. 4. Graphical representation of a data generating system where the measured variable $w_{i}$ is excited through an external excitation signal $r_{m}$.

with $\Upsilon(z)$ monic, proper, stable and minimum-phase. This implies that $r_{1}$ is modelled as a filtered white noise $e_{1}$. $\Upsilon_{0}(q)$ can be estimated as a noise model in a prediction error method with no measured input. From the estimated model $\Upsilon\left(q, \hat{\theta}_{N}\right)$ estimates of the transfers $G_{21}^{0}$ and possibly $G_{12}^{0}$ can then be calculated in a second step.

Typical conditions for consistency of the estimate of the module transfer $G_{21}^{0}$ are basically the same as the conditions that are required for the direct method, i.e. sufficient excitation of the closed-loop, and an absence of algebraic loops. One of the points of difference is that the joint-IO method also allows to estimate to feedback path represented by $G_{12}^{0}$.

A generalization of this method to the dynamic network case is obtained by enlarging the set of node signals that is taken into account in the first step of the procedure.

In the next sections it will be shown how the direct, the two-stage and the joint-IO methods can be used to obtain consistent estimates of $G_{j i}^{0}$. However first a few network properties will be presented that will facilitate the analysis of the identification methods.

\section{Network properties}

It will be natural to talk about paths and loops in a network. A path from $i$ to $j$ will be understood to mean that there are transfer functions such that $G_{j n_{1}} G_{n_{1} n_{2}} \cdots G_{n_{k} i}$ is nonzero. A loop is a path that has the same starting and ending node. The length of a path is the number of transfer functions in the product.

One of the properties of a dynamic network is the possible occurrence of algebraic loops, i.e. loops for which the transfer function has a direct feed-through term. For analyzing the properties of direct identification algorithms it is attractive to be able to characterize these loops. In a network with modules that are restricted to be strictly proper transfer functions, algebraic loops will not occur but in the situation that we consider here they are not excluded.

Lemma 3 Consider a dynamic network that satisfies Assumption 1, with transfer matrix $G^{0}(2 a)$. Let $\mathcal{G}_{a b}^{0}$ be the $(a, b)$ th entry of $\left(I-G^{0}\right)^{-1}$. Then $\mathcal{G}_{a b}^{0}$, has a delay if every path from $b$ to a has a delay. Moreover, $\mathcal{G}_{a b}^{0}=0$ if there is no path from $b$ to $a$.

The proof of the Lemma is included in the appendix. The next proposition shows that the dynamic network can be rewritten in a classical feedback structure by denoting one particular $w_{j}$ to be as "output signal". Any node signal can serve this purpose. This equivalent structure will facilitate the understanding and analysis of the several identification results.

Proposition 1 Consider a dynamic network that satisfies Assumption 1, and select one particular node signal $w_{j}$ to be referred to as "output". Classify the remaining signals $w_{i}, i \in \mathcal{D}$ with $\mathcal{D}:=\{1, \cdots L\} \backslash\{j\}$ as inputs, denoted as

$$
w_{\mathcal{D}}=\left[\begin{array}{lll}
w_{k_{1}} & w_{k_{2}} \cdots
\end{array}\right]^{T}, \quad k_{*} \in \mathcal{D} .
$$

The vectors $r_{\mathcal{D}}$ and $v_{\mathcal{D}}$ and defined analogously.

Let $G_{j \mathcal{D}}^{0}$ denote the row vector $\left[G_{j k_{1}}^{0} G_{j k_{2}}^{0} \cdots\right], k_{*} \in \mathcal{D}$, let $G_{\mathcal{D} j}^{0}$ denote the column vector $\left[G_{k_{1} j}^{0} G_{k_{2} j}^{0} \cdots\right]^{T}, k_{*} \in \mathcal{D}$, and let $G_{\mathcal{D} \mathcal{D}}^{0}$ denote the corresponding matrix.

The measured node signals $\left\{w_{k}\right\}_{k=1, \cdots L}$ are equivalently described by the feedback connection structure as indicated in Figure 5, with $w_{j}$ interpreted as output, and $w_{\mathcal{D}}$ as input, determined by

$$
\begin{aligned}
w_{j} & =G_{j \mathcal{D}}^{0}(q) w_{\mathcal{D}}+r_{j}+v_{j} \\
w_{\mathcal{D}} & =\breve{G}_{\mathcal{D} j}^{0}(q) w_{j}+\breve{G}^{v}(q) v_{\mathcal{D}}+\breve{G}^{r}(q) r_{\mathcal{D}},
\end{aligned}
$$

with

$$
\begin{aligned}
\breve{G}^{v} & =\breve{G}^{r}=\left(I-G_{\mathcal{D D}}^{0}\right)^{-1} \\
\breve{G}_{\mathcal{D} j}^{0} & =\left(I-G_{\mathcal{D} \mathcal{D}}^{0}\right)^{-1} G_{\mathcal{D} j}^{0}
\end{aligned}
$$

and with proper square transfer matrices $\breve{G}^{v}, \breve{G}^{r} \in$ $\mathbb{R}^{(L-1) \times(L-1)}(z)$, and proper transfer vector $\breve{G}_{\mathcal{D} j}^{0} \in$ $\mathbb{R}^{(L-1) \times 1}(z)$.

Proof Using the introduced notation the network equations (2b) can be written as

$$
\left[\begin{array}{c}
w_{j} \\
w_{\mathcal{D}}
\end{array}\right]=\left[\begin{array}{cc}
0 & G_{j \mathcal{D}}^{0} \\
G_{\mathcal{D} j}^{0} & G_{\mathcal{D} \mathcal{D}}^{0}
\end{array}\right]\left[\begin{array}{l}
w_{j} \\
w_{\mathcal{D}}
\end{array}\right]+\left[\begin{array}{c}
r_{j}+v_{j} \\
r_{\mathcal{D}}+v_{\mathcal{D}}
\end{array}\right]
$$

leading to the network equations:

$$
\begin{aligned}
w_{j} & =G_{j \mathcal{D}}^{0} w_{\mathcal{D}}+r_{j}+v_{j} \\
w_{\mathcal{D}} & =\left(I-G_{\mathcal{D} \mathcal{D}}^{0}\right)^{-1} G_{\mathcal{D} j}^{0} w_{j}+\left(I-G_{\mathcal{D} \mathcal{D}}^{0}\right)^{-1}\left(r_{j}+v_{j}\right)
\end{aligned}
$$

provided that the inverse $\left(I-G_{\mathcal{D} \mathcal{D}}^{0}\right)^{-1}$ exists and is proper. This is guaranteed by condition 1 of Assumption 1 . 
One of the important observations from the presented feedback structure is that the disturbance/excitation terms that directly affect the "output" $w_{j}$, do not appear as disturbance/excitation signals directly acting on the "input signals" $w_{\mathcal{D}}$; they only contribute to these inputs through the "feedback" operation $\breve{G}_{\mathcal{D} j}^{0}$.

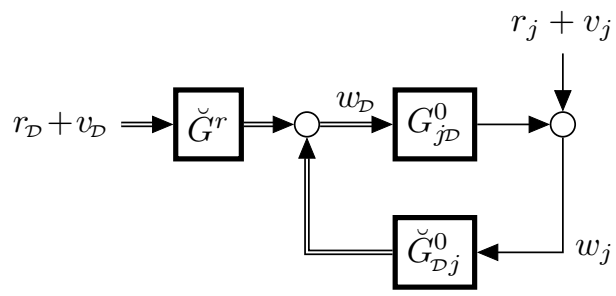

Fig. 5. Closed Loop representation of $w_{j}$.

\section{Direct method for general network topology}

The direct method for closed-loop identification can rather simply be generalized to the situation of dynamic networks. To this end we consider the one-step ahead predictor that was formulated in (4). The principal choice that has to be made is the set of input signals $x_{k} \in \mathcal{X}$ that has to be taken into account in the predictor. If the module transfer function $G_{j i}^{0}$ needs to be identified it is tempting to choose $w_{i}$ as input for the predictor. However in most cases this will lead to biased results of the estimates due to the fact that other (neglected) input signals will affect the output also. Therefore the most safe situation is to choose in the predictor all inputs that have a direct link to the output $w_{j}$, i.e. $\mathcal{X}=\mathcal{N}_{j}$, leading to the predictor:

$$
\begin{array}{r}
\hat{w}_{j}(t \mid t-1 ; \theta)=H_{j}^{-1}(q, \theta)\left(\sum_{k \in \mathcal{N}_{j}} G_{j k}(q, \theta) w_{k}(t)+r_{j}(t)\right) \\
+\left(1-H_{j}^{-1}(q, \theta)\right) w_{j}(t) .
\end{array}
$$

For this predictor the following result is obtained.

Proposition 2 Consider a dynamic network that satisfies Assumption 1, and consider a direct prediction error identification according to (5) with predictor (13). Then the module transfer functions $G_{j k}^{0}, k \in \mathcal{N}_{j}$ as well as $H_{j}^{0}$ are estimated consistently under the following conditions:

(a) The noise $v_{j}$ is uncorrelated to all reference signals.

(b) The noise $v_{j}$ is uncorrelated to all noise signals $v_{k}$, $k \in \mathcal{V}_{j} \backslash\{j\}$.

(c) For both the network and the parametrized model, every loop through node $j$ has a delay.

(d) The spectral density of $\left[\begin{array}{llll}w_{j} & w_{n_{1}} & \cdots & w_{n_{n}}\end{array}\right]^{T}, n_{*} \in \mathcal{N}_{j}$, denoted as $\Phi_{j, \mathcal{N}_{j}}(\omega)$ is positive definite for $\omega \in[-\pi, \pi]$.

(e) The system is in the model set, i.e. there exists a $\theta^{0}$ such that $G_{j k}\left(z, \theta^{0}\right)=G_{j k}^{0}(z)$ for all $k \in \mathcal{N}_{j}$, and $H_{j}\left(z, \theta^{0}\right)=H_{j}^{0}(z)$.
The proof is added in the appendix.

Note that in the considered situation all transfers $G_{j k}^{0}, k \in$ $\mathcal{N}_{j}$ need to be estimated simultaneously in order for the result to hold, and that the dynamics of noise source $v_{j}$ needs to be modeled correctly through a noise model $H_{j}$. Note also that both the noise signal $v_{j}$ and the probing signal $r_{j}$ provide excitation to the loop that is going to be identified. The excitation condition (d) is a rather generic condition for informative data [15]. A further specification for particular finite dimensional model structures can most likely be made along the results for classical feedback loops as developed in [12].

Whereas in classical closed loop identification with the direct method there is a condition on absence of algebraic loops in the full feedback system ([21]), this is further specified here in condition (c) by limiting that condition to only apply to the output signal that is considered for identification.

Remark 1 In the Proposition above the predictor that is used employs all possible inputs that directly connect to the output signal $w_{j}$. If some of these transfers are known already, e.g. they could be controllers with known dynamics, then the result above can simply be generalized to the predictor

$$
\begin{aligned}
\hat{w}_{j}(t, \theta)= & H_{j}^{-1}(q, \theta)\left(\sum_{k \in \mathcal{N}_{j} \backslash \mathcal{K}_{j}} G_{j k}(q, \theta) w_{k}(t)+\right. \\
& \left.+\sum_{k \in \mathcal{K}_{j}} G_{j k}^{0}(q) w_{k}(t)+r_{j}(t)\right)+ \\
& \quad+\left(1-H_{j}^{-1}(q, \theta)\right) w_{j}(t),
\end{aligned}
$$

leading to consistent estimates of the transfers $G_{j k}^{0}, k \in$ $\mathcal{N}_{j} \backslash \mathcal{K}_{j}$, while in the formulation of the conditions of Proposition 2 , the set $\mathcal{N}_{j}$ is replaced by the set $\mathcal{N}_{j} \backslash \mathcal{K}_{j}$.

Next, an algorithm for checking Condition (c) will be presented. The other conditions are straightforward to check and do not need an algorithm. Recall that the matrix $A_{d}$ is the adjacency matrix with $d$ 's in the entries with strictly proper module transfer functions, and 1's in the entries with proper module transfer functions (see Section 2.2).

\section{Algorithm 1 Check if all loops through node $j$ have a delay}

(1) Evaluate $A_{d}^{\ell}$ for $\ell=1, \ldots, L$ using the multiplication and addition rules defined in Section 2.2;

(2) If for any considered power $\ell$ entry $(j, j)$ equals 1 , Condition (c) is not met.

Example 1 If we apply the result of the direct method to the network example of Figure 6, it appears that the direct method can be applied to each of the nodes $w_{1}, \cdots w_{5}$. Note that in this scheme $G_{43}^{0}=1$. The blue-colored transfers $G_{15}^{0}, G_{32}^{0}, G_{54}^{0}, G_{45}^{0}$ can be identified by SISO predictors, 
using only a single input in the predictor, provided that appropriate conditions are satisfied on excitation and absence of algebraic loops. The transfers $G_{21}^{0}$ and $G_{23}^{0}$ can only be estimated simultaneously in a MISO predictor, employing both $w_{1}$ and $w_{3}$ as inputs and $w_{2}$ as output. Under the condition that a delay is present in the loops $\left(G_{32}^{0} G_{23}^{0}\right)$ and $\left(G_{54}^{0} G_{32}^{0} G_{21}^{0} G_{15}^{0}\right)$ and by the use of an appropriate model set that includes accurate noise modelling, the transfers $G_{21}^{0}$ and $G_{23}^{0}$ can be estimated consistently. In Figure 6 they are indicated in red.

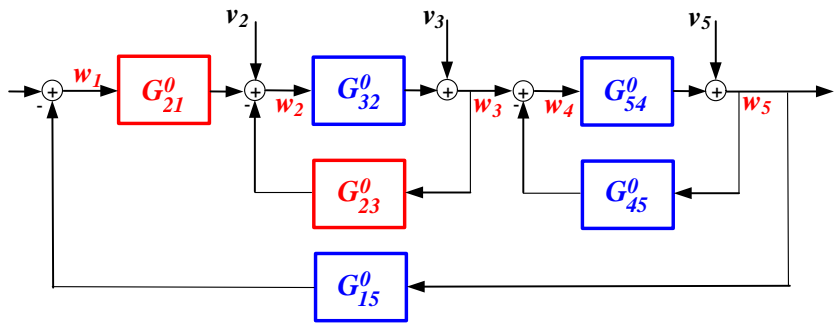

Fig. 6. Dynamic network with 5 node signals, of which 2 (red-colored) transfer functions $G_{21}^{0}$ and $G_{23}^{0}$ can be consistently identified with the direct method using a MISO predictor. The blue-colored transfer functions can be identified with SISO predictors.

It is clear that in large and complex networks only under very limited circumstances, a SISO predictor, i.e. having only the input $w_{i}$ present in the predictor, suffices to consistently identify the transfer $G_{j i}^{0}$.

Remark 2 It is not always necessary to include all $w_{k}$, $k \in \mathcal{N}_{j}$ as input in the predictor. For instance consider the case shown in Fig. 7a. Suppose that the objective is to obtain consistent estimates of $G_{21}^{0}$. According to Proposition 2 both $w_{1}$ and $w_{3}$ must be included as inputs in the predictor. However, from the figure, it can be seen that $w_{3}$ only acts as a (uncorrelated) disturbance on $w_{2}$, and does not need to be modelled for consistent estimation of $G_{21}^{0}$. This idea is illustrated in Fig. $7 b$ where $\tilde{v}_{2}=v_{2}+w_{3}$.

Further analysis of this is beyond the scope of the current paper and will be presented elsewhere.

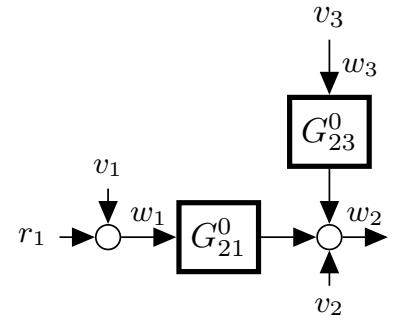

(a)

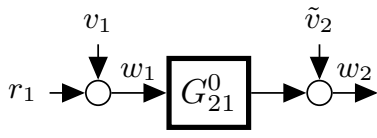

Fig. 7. Example of a system where not all $w_{k}, k \in \mathcal{N}_{j}$ need to be included as inputs in the predictor ( $w_{3}$ can just be considered as an uncorrelated disturbance).

Note that for using signal $w_{j}$ as an output, it is not strictly necessary that a noise source $v_{j}$ is present. This special case is considered in the next Corollary.
Corollary 1 Consider the situation of Proposition 2. If the noise source $v_{j}$ is not present. Then the module transfer functions $G_{j i}^{0}, i \in \mathcal{N}_{j}$ can be estimated consistently, under the conditions of Proposition 2, where the excitation condition $(d)$ is replaced by:

(d) The spectrum of $\left[w_{n_{1}} \cdots w_{n_{\mathfrak{n}}}\right]^{T}, n_{*} \in \mathcal{N}_{j}, \Phi_{\mathcal{N}_{j}}(\omega)$ is positive definite for $\omega \in[-\pi, \pi]$,

the delay condition (c) is removed, and the noise model is fixed to 1, thereby focussing condition (e) on the module transfer property only.

Proof The same procedure as the proof of Proposition 2 can be followed starting with (B.3) and plugging in $v_{j}=0$, $H(\theta)=1$, and $\sigma_{e_{j}}^{2}=0$.

\section{Two-stage identification for general network topology}

The two-stage method for closed-loop identification as described in Section 3.3 follows a different approach than the direct method. It explicitly utilizes the presence of measurable external excitation signals, and has the potential to consistently identify module transfers without the necessity to consistently identify noise models also. Based on the scheme depicted in Figure 4 we pursue the following strategy in an attempt to consistently identify the module transfer $G_{j i}^{0}$.

\section{Algorithm 2 (Two-stage SISO model)}

(1) Select a set of measured excitation signals $\left\{r_{m}\right\}$, with $m \in \mathcal{R}_{i s} \subseteq \mathcal{R}_{i}$, each of them correlated with $w_{i}$.

(2) On the basis of measured signals $\left\{r_{m}\right\}, m \in \mathcal{R}_{\text {is }}$ and $w_{i}$, determine $w_{i}^{\left(\mathcal{R}_{i s}\right)}$.

(3) Construct the signal

$\tilde{w}_{j}(t)=w_{j}(t)-\sum_{k \in \mathcal{K}_{j}} G_{j k}^{0}(q) w_{k}(t)-r_{j}(t)$,

i.e. correct $w_{j}$ with all known terms;

(4) Identify the transfer function $G_{j i}^{0}$ on the basis of a predictor model with prediction error

$$
\varepsilon_{j}(t, \theta)=H_{j}(q, \eta)^{-1}\left[\tilde{w}_{j}(t)-G_{j i}(q, \theta) w_{i}^{\left(\mathcal{R}_{i s}\right)}(t)\right]
$$

using measured signals $\tilde{w}_{j}$ and $w_{i}^{\left(\mathcal{R}_{i s}\right)}$, an identification criterion (5), and where $H_{j}$ is a fixed noise model or parametrized independently of $\theta$.

For this algorithm the following result can be obtained:

Proposition 3 Consider a dynamic network that satisfies Assumption 1. Then the module transfer function $G_{i i}^{0}$ can be consistently estimated with algorithm 2 if the following conditions are satisfied:

(a) The set $\mathcal{R}_{\text {is }}$ is non-empty.

(b) The external excitation signals $r_{m} m \in \mathcal{R}_{\text {is }}$ are uncorrelated to all noise signals $v_{k}, k \in\left\{j, \mathcal{U}_{j}^{i}\right\}$; 
(c) The signal $w_{i}^{\left(\mathcal{R}_{i s}\right)}$ is persistently exciting of a sufficiently high order ${ }^{2}$

(d) All node signals $w_{k}, k \in \mathcal{U}_{j}^{i}, k \neq i$, are uncorrelated to all $r_{m}, m \in \mathcal{R}_{i s}$.

(e) The module transfer function $G_{j i}^{0}$ is in the model set, i.e. there exists a parameter $\theta^{0}$ such that $G_{j i}\left(q, \theta^{0}\right)=$ $G_{j i}^{0}(q)$.

Proof: Note that $w_{j}$ can be expressed as

$$
\begin{aligned}
w_{j}(t)= & G_{j i}^{0}(q) w_{i}(t)+\sum_{k \in \mathcal{K}_{j}} G_{j k}^{0}(q) w_{k}(t) \\
& +\sum_{k \in \mathcal{U}_{j}^{i}} G_{j k}^{0}(q) w_{k}(t)+r_{j}(t)+v_{j}(t) \\
= & G_{j i}^{0}(q) w_{i}(t)+p_{j}(t)+s_{j}(t)+v_{j}(t)
\end{aligned}
$$

where $p_{j}$ reflects the contributions of all signals $G_{j k}^{0}(q) w_{k}$ that are known because of the fact that the dynamics $G_{j k}^{0}$ are known, as well as $r_{j}(t)$; and $s_{j}(t)$ similarly reflects the contributions of all signals $G_{j k}^{0}(q) w_{k}$ that are unknown, because the dynamics $G_{j k}^{0}$ is unknown.

Subsequently

$$
w_{j}(t)-p_{j}(t)=G_{j i}^{0}(q) w_{i}(t)+s_{j}(t)+v_{j}(t)
$$

with the left hand side being a known signal.

Condition (b) together with the fact that by construction all $r_{m}, m \in \mathcal{R}_{i s}$ are correlated to $w_{i}$, guarantee that $w_{i}$ can be decomposed as $w_{i}=w_{i}^{\left(\mathcal{R}_{i s}\right)}+w_{i}^{\left(\perp \mathcal{R}_{i s}\right)}$. Then,

$$
w_{j}-p_{j}=G_{j i}^{0}(q)\left(w_{i}^{\left(\mathcal{R}_{i s}\right)}+w_{i}^{\left(\perp \mathcal{R}_{i s}\right)}\right)+s_{j}+v_{j} .
$$

Conditions (b) and (d) guarantee that the signal $s_{j}$ is uncorrelated to all $r_{m}, m \in \mathcal{R}_{i s}$. And by condition (b) the noise $v_{j}$ is uncorrelated to all $r_{m}, m \in \mathcal{R}_{i s}$, while $w_{i}^{\left(\perp \mathcal{R}_{i s}\right)}$ is uncorrelated to all $r_{m}, m \in \mathcal{R}_{i s}$ by construction.

As a result a prediction error identification on the basis of input $w_{i}^{\left(\mathcal{R}_{i s}\right)}$ and output $w_{j}-p_{j}$ will provide a consistent estimate of $G_{j i}^{0}$, provided that the input signal $w_{i}^{\left(\mathcal{R}_{i s}\right)}(t)$ is persistently exciting of a degree at least equal to the number of parameters in $G_{j i}(q, \theta)$, see the classical conditions on consistency of prediction error estimates in [15].

Note that as an alternative for the two-stage algorithm, also an IV estimator could have been used, using $r_{m}$ as instrument, $w_{i}$ as input and $w_{j}-p_{i}$ as output, leading to the same consistency result, [13].

Next question is how to check whether the conditions of

\footnotetext{
2 Within the classical prediction error framework [15], the reconstructed signal $w_{i}^{\left(\mathcal{R}_{i s}\right)}(t)$ will need to be persistently exciting of an order at least equal to the number of unknown parameters that is estimated in $G_{j i}(q, \theta)$.
}

Proposition 3 are satisfied. Both the appropriate construction of the set $\mathcal{R}_{i s}$ and Condition (d) can be checked mainly on the basis of the adjacency matrix $A$ of the network.

\section{Algorithm 3}

Check for candidate reference signals to be correlated to $w_{i}$ :

(1) Evaluate element $(i, m)$ of $A^{\ell}$ for $\ell=1, \cdots L$;

(2) If for any considered power $\ell$ this element is non-zero, then the reference signal $r_{m}$ qualifies as a candidate excitation source that excites the input $w_{i}{ }^{3}$

Check whether all $w_{k}, k \in \mathcal{U}_{j}^{i}$ are uncorrelated to all $r_{m}$, $m \in \mathcal{R}_{\text {is }}$ (check whether there is no path from $m$ to $k$ ):

(1) Evaluate $A^{\ell}$ for $\ell=1, \ldots, L$;

(2) For all $k \in \mathcal{U}_{j}^{i}, k \neq i$, check whether the entries $(k, m)$ of $A^{\ell}$ are zero for all powers $\ell$.

Example 2 Consider the dynamic network from Example 1, depicted in Figure 8. When applying the conditions of Proposition 3 it appears that the blue-colored transfers, $G_{32}^{0}, G_{54}^{0}$, $G_{15}^{0}$ and $G_{45}^{0}$ can be consistently identified with the twostage approach presented in this section. These four transfers satisfy the conditions that their inputs are correlated to $r_{1}$, while their outputs do not include non-modelled terms that are correlated with $r_{1}$.

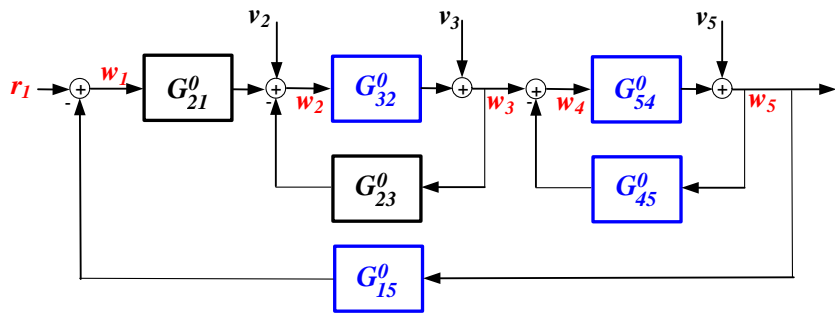

Fig. 8. Dynamic network with 5 node signals, of which 4 (bluecolored) transfer functions can be consistently identified with the two-stage method presented of Algorithm 2.

Note that the transfers $G_{21}^{0}$ and $G_{23}^{0}$ do not satisfy the conditions of the Proposition because there are unknown contributions to $w_{2}$ that are correlated to $r_{1}$.

Actually the conditions that are formulated for Proposition 3 are very restrictive and it may be very well possible that even in case of networks that have several external excitation signals present, there is no choice of $\mathcal{R}_{i s}$ possible that satisfies the conditions. Additionally, by limiting attention to SISO predictors, i.e. by only considering $w_{i}$ as input in the predictor, the effect of all other inputs that affect $w_{j}$ will

\footnotetext{
${ }^{3}$ In the case that $\sum_{\ell=1}^{N}\left[A^{\ell}\right]_{i m}>1$, there is a hypothetical option that different path connections cancel each other. Since the actual correlation between $r_{m}$ and $w_{i}$ always needs to be checked, this situation will not be dealt with separately.
} 
be modelled as noise and therefore will increase the variance of the parameter estimate. An alternative reasoning, that matches the situation of the direct method, is then to extend the predictor to a MISO format, as reflected in the following algorithm.

\section{Algorithm 4 (Two-stage MISO model)}

(1) Select a set of measured excitation signals $\left\{r_{m}\right\}$, with $m \in \mathcal{R}_{i s} \subseteq \mathcal{R}_{i}$, each of them correlated with $w_{i}$.

(2) Determine the set of node signals $w_{k}, k \in\left\{\mathcal{U}_{j}^{i}, i\right\}$ that is correlated to any of the excitation signals $\left\{r_{m}\right\}$, with $m \in \mathcal{R}_{i s}$. Denote this set as $\left\{w_{k}\right\}, k \in \mathcal{U}_{i s}$.

(3) Determine $\left.w_{k}^{\left(\mathcal{R}_{i s}\right.}\right)$, for $k \in \mathcal{U}_{i s}$.

(4) Construct the signal $\tilde{w}_{j}(t)=w_{j}(t)-\sum_{k \in \mathcal{K}_{j}} G_{j k}^{0}(q) w_{k}(t)-r_{j}(t)$, i.e. correct $w_{j}$ with all known terms;

(5) Identify the transfers $G_{j k}^{0}, k \in \mathcal{U}_{i s}$ on the basis of a predictor model with prediction error

$$
\left.\varepsilon_{j}(t, \theta)=H_{j}(q, \eta)^{-1}\left[\tilde{w}_{j}(t)-\sum_{k \in \mathcal{U}_{j s}} G_{j k}(q, \theta) w_{k}^{\left(\mathcal{R}_{i s}\right.}\right)(t)\right]
$$

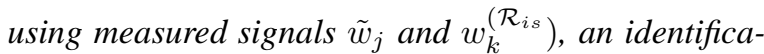
tion criterion (5), and where $H_{j}$ is a fixed noise model or parametrized independently of $\theta$.

For this algorithm the following result can be obtained:

Proposition 4 Consider a dynamic network that satisfies Assumption 1. Then the module transfer function $G_{j i}^{0}$ can be consistently estimated with algorithm 4 if the following conditions are satisfied:

(a) The set $\mathcal{R}_{\text {is }}$ is non-empty.

(b) The external excitation signals $r_{m}, m \in \mathcal{R}_{\text {is }}$ are uncorrelated to noise signals $v_{k}, k \in\left\{j, \mathcal{U}_{j}^{i}\right\}$.

(c) The power spectral density of $\left[\begin{array}{llll}\left.w_{n_{1}}^{\left(\mathcal{R}_{i s}\right.}\right) & \cdots & \left.w_{n_{\mathfrak{n}}}^{\left(\mathcal{R}_{i s}\right.}\right)\end{array}\right]^{T}$, $n_{*} \in \mathcal{U}_{i s}$ is positive definite for $\omega \in[-\pi, \pi]$.

(d) The module transfers $G_{j k}^{0}$ are in the model set, i.e. there exists a parameter $\theta^{0}$ such that $G_{j k}\left(q, \theta^{0}\right)=G_{j k}^{0}(q)$ for all $k \in \mathcal{U}_{i s}$.

Under the considered conditions, all model transfer functions $G_{j k}^{0}, k \in \mathcal{U}_{i s}$ are estimated consistently.

Proof: The proof follows along similar lines as the proof of Proposition 3 with appropriate change of notation.

Example 3 Returning now to the situation of Example 2, it can be observed that with Algorithm 4, the remaining module transfers $G_{21}^{0}$ and $G_{23}^{0}$ can be identified by using a MISO predictor with inputs $w_{1}$ and $w_{3}$ and output $w_{2}$. The external excitation signal $r_{1}$ excites both inputs. It only has to be checked whether this excitation is sufficiently informative. Adding a second excitation signal could be helpful in this respect.
Moving from a SISO to a MISO predictor further increases the complexity of the identification procedure, in terms of number of models and parameters to be estimated. However it also can substantially reduce the variance of the estimates by improving the effective signal-to-noise ratio in the output. The choice for which inputs to use in the predictor, and which external excitation signals to project upon, leaves more freedom here to choose from. This aspect is further developed elsewhere [6].

Although in the framework of this paper, we are dealing with noise-free measurements of node signals $w_{j}$, it has to be noted that the two-stage method can simply be generalized to deal with the situation of having measurement noise on the node signals also. This is caused by the property that measurement noise will disappear when the measured node signals will be projected upon external excitation signals.

\section{Extension of two-stage method with reconstructible noise signals}

Whereas in the two-stage method measured external excitation signals serve as a basis for removing noise influences from input signals by way of projection, a similar mechanism can be realized under particular circumstances by noise signals. Consider the situation that somewhere in the network there is a noise signal $v_{m}$ present, that can be reconstructed on the basis of measured signals and known transfers, and that provides excitation for the node signal $w_{i}$ that is an input to the transfer function $G_{j i}^{0}$. Then a reasoning that is completely similar to the two-stage method of the previous section can be applied by treating this reconstructible noise signal as an external excitation signal.

The situation is depicted in Figure 9, where noise signal $v_{m}$ is reconstructible if all transfers $G_{m k}^{0}, k \in \mathcal{N}_{m}$ are known. Then signal $x_{m}$ can be calculated and $v_{m}$ can be reconstructed according to $v_{m}=w_{m}-x_{m}$. From this moment onwards $v_{m}$ can act as as an external excitation signal that can be used in both the SISO and MIMO predictor of the two-stage method.

An algorithm for checking whether a noise signal is reconstructible is easily generated. For every index $m \in \mathcal{V}$ : check if $\mathcal{K}_{m}=\mathcal{N}_{m}$. If so, $v_{m}$ qualifies as a reconstructible noise signal. Algorithms for checking whether $v_{m}$ satisfies the appropriate correlation properties with respect to the inputs $w_{i}$ and $w_{k}, k \in \mathcal{N}_{j}$ are equivalent to the ones provided in the previous section.

Example 4 If we consider the network example of Figure 8, it appears that both $v_{3}$ and $v_{5}$ qualify as a reconstructible noise signal, provided that the transfers $G_{32}^{0}$ and $G_{54}^{0}$ are known a priori. However in the considered situation none of the remaining transfer functions satisfies the other condition of Proposition 3 that the outputs should not be disturbed by unknown terms that are correlated to the (reconstructible) noise source. 


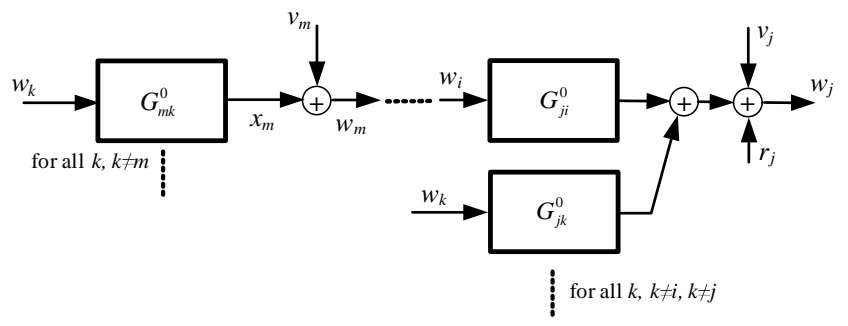

Fig. 9. Single node in a network structure, where the input $w_{i}$ is excited through a reconstructed noise signal $v_{m}$.

However if we remove the outer loop connection $G_{15}^{0}$, as depicted in Figure 10, then $G_{23}^{0}$ can be identified consistently through reconstructible noise signal $v_{3}$ if $G_{32}^{0}$ is known. In Figure 10 this transfer is indicated in red. Similarly, using a two-input predictor the two-stage method can now be applied to node signal $w_{2}$ with inputs $w_{1}, w_{3}$ and external excitation signals $r$ and $v_{3}$.

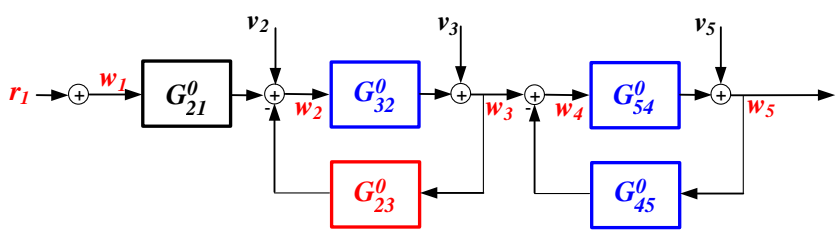

Fig. 10. Dynamic network with 5 node signals, of which 1 (redcolored) transfer function can be consistently identified with the two-stage method presented in this section 7 based on reconstructed noise signals.

The special phenomenon with reconstructible noise signals, is the appealing mechanism that a noise signal with varianceincreasing effects on the model estimates, by the use of a prior knowledge of particular module transfers, can be turned into an external excitation signal that reduces the variance of the estimates.

\section{Joint IO method for general network topology}

Also the joint IO method can be generalized to the situation of dynamic networks. As with the other methods presented before, we will focus on a particular node signal $w_{j}$, for which we intend to identify the module transfer $G_{j i}^{0}$. When isolating the two node signals $w_{i}$ and $w_{j}$, and modeling the vector process $\left(w_{j}^{T}, w_{i}^{T}\right)^{T}$ as the output of a stationary stochastic process, it is very unlikely that the resulting process will allow to determine consistent estimates of $G_{j i}^{0}$, if the two node signals are part of a complex network topology. Like in the direct method, we have to extend the number of node signals that we take into account.

Consider the following partition of measured variables: $w=$ $\left\{w_{j}, w_{\mathcal{N}_{j}}, w_{z_{j}}\right\}$ where $\mathcal{N}_{j}$ has the usual meaning, and $\mathcal{Z}_{j}$ is a set of all remaining variables. In the subsequent text the subscript $j$ will be dropped from $\mathcal{N}_{j}$ and $\mathcal{Z}_{j}$ for notational simplicity. Using these partitions the data generating system can be written as follows ${ }^{4}$ :

$$
\left[\begin{array}{c}
w_{j} \\
w_{\mathcal{N}} \\
w_{\mathcal{Z}}
\end{array}\right]=\left[\begin{array}{ccc}
0 & G_{j \mathcal{N}}^{0} & 0 \\
G_{\mathcal{N} j}^{0} & G_{\mathcal{N N}}^{0} & G_{\mathcal{N Z}}^{0} \\
G_{\mathcal{Z} j}^{0} & G_{\mathcal{Z N}}^{0} & G_{\mathcal{Z Z}}^{0}
\end{array}\right]\left[\begin{array}{l}
w_{j} \\
w_{\mathcal{N}} \\
w_{\mathcal{Z}}
\end{array}\right]+\left[\begin{array}{c}
v_{j} \\
v_{\mathcal{N}} \\
v_{\mathcal{Z}}
\end{array}\right]
$$

where the vector $v_{\mathcal{N}}$ is defined as vector of the same dimension as $w_{\mathcal{N}}$ with either $v_{i}, i \in \mathcal{N}$ present or 0 if the particular node signal does not contain an external disturbance (or excitation) signal. The vector $v_{\mathcal{z}}$ is similarly defined.

In the joint-IO method we first model the measured signals $\left(w_{j}, w_{\mathcal{N}}\right)$ as output of a stationary stochastic process. Next an estimate of the module transfer function $G_{j i}^{0}$ is extracted from this previously estimated noise model.

First we are going to formalize the properties of the vector process $\left(w_{j}, w_{\mathcal{N}}\right)$ in the next Lemma.

Lemma 4 The node signals $w_{j}, w_{\mathcal{N}}$ satisfy the following representation:

$$
\begin{aligned}
& {\left[\begin{array}{l}
w_{j} \\
w_{\mathcal{N}}
\end{array}\right] }=W^{0}\left[\begin{array}{l}
e_{j} \\
\tilde{e}_{\mathcal{N}}
\end{array}\right] \text { with } \\
& W^{0}:=\left[\begin{array}{ll}
W_{j j}^{0} & W_{j \mathcal{N}}^{0} \\
W_{\mathcal{N} j}^{0} & W_{\mathcal{N N}}^{0}
\end{array}\right]=\left[\begin{array}{cc}
\breve{G}_{j j}^{0} H_{j}^{0} & G_{j \mathcal{N}}^{0} \breve{G}_{\mathcal{N N}}^{0} \tilde{H}_{\mathcal{N}}^{0} \\
\breve{G}_{\mathcal{N N}}^{0} \tilde{G}_{\mathcal{N} j}^{0} H_{j}^{0} & \breve{G}_{\mathcal{N N}}^{0} \tilde{H}_{\mathcal{N}}^{0}
\end{array}\right]
\end{aligned}
$$

with $e_{j}$ and $\tilde{e}_{\mathcal{N}}$ uncorrelated white noise processes, and where

$$
\begin{aligned}
\breve{G}_{j j}^{0} & =\left(1-G_{j \mathcal{N}}^{0}\left(I-\tilde{G}_{\mathcal{N N}}^{0}\right)^{-1} \tilde{G}_{\mathcal{N} j}^{0}\right)^{-1} \\
\breve{G}_{\mathcal{N N}}^{0} & =\left(I-\tilde{G}_{\mathcal{N N}}^{0}-\tilde{G}_{\mathcal{N} j}^{0} G_{j \mathcal{N}}^{0}\right)^{-1} \\
\tilde{G}_{\mathcal{N N}}^{0} & =G_{\mathcal{N N}}^{0}+G_{\mathcal{N Z}}^{0}\left(I-G_{\mathcal{Z Z}}^{0}\right)^{-1} G_{\mathcal{Z N}}^{0} \\
\tilde{G}_{\mathcal{N} j}^{0} & =G_{\mathcal{N} j}^{0}+G_{\mathcal{N Z}}^{0}\left(I-G_{\mathcal{Z Z}}^{0}\right)^{-1} G_{\mathcal{Z} j}^{0},
\end{aligned}
$$

and $\tilde{H}_{\mathcal{N}}^{0}$ is the monic, stable minimum-phase spectral factor of the stochastic process $v_{\mathcal{N}}+G_{\mathcal{N Z}}^{0}\left(I-G_{\mathcal{Z Z}}^{0}\right)^{-1} v_{\mathcal{Z}}$.

If the matrix $W^{0}$ in (17) is available (or an estimate thereof) then it is possible to reconstruct $G_{j \mathcal{N}}^{0}$ and $H_{j}^{0}$, according to:

$$
\begin{aligned}
G_{j \mathcal{N}}^{0} & =W_{j \mathcal{N}}^{0} W_{\mathcal{N N}}^{0}{ }^{-1} \\
H_{j}^{0} & =W_{j j}^{0}-W_{j \mathcal{N}}^{0} W_{\mathcal{N N}}^{0}{ }^{-1} W_{\mathcal{N} j}^{0} .
\end{aligned}
$$

An estimate of $W^{0}$ can be obtained by estimating a noise model which whitens the stochastic processes which generate the data. In particular, the output of the stochastic process is $w(t)=\left[w_{j}(t) w_{\mathcal{N}}(t)\right]^{T}$, and the input is $e(t)=$

\footnotetext{
4 Since in the Joint IO method no explicit use is made of measured external excitation signals, we assume that $r$-signals are not present, and that all external excitation originates from noisy $v$ signals.
} 
$\left[e_{j}(t) \tilde{e}_{\mathcal{N}}(t)\right]^{T}$. The one-step ahead prediction error is:

$$
\varepsilon(t, \theta)=w(t)-\hat{w}(t \mid t-1 ; \theta)=W^{-1}(\theta) W^{0} e(t)
$$

and $W(\theta)$ is the parametrized noise model.

Whereas in the standard prediction error situation it can be assumed that $W^{0}$ and $W(\theta)$ are monic, stable minimumphase transfer function matrices, this condition is infeasible here, as $W^{0}$ is not necessarily monic. As a result, a dedicated parametrization of $W(\theta)$ needs to be chosen, in particular for the modelling of the constant feedthrough term $W^{0}(\infty)$, to be parametrized by $W(\infty, \theta)$.

The algorithm for the joint-IO method now becomes:

\section{Algorithm 5 (Joint-IO method)}

(1) Choose a parametrization $W(\theta)$ of $W^{0}$;

(2) Determine $\hat{\theta}_{N}$ by minimizing the sum of squared prediction errors, $V_{N}(\theta)=\frac{1}{N} \sum_{t=0}^{N-1} \varepsilon^{T}(t, \theta) \varepsilon(t, \theta)$.

(3) Calculate $\hat{G}_{j \mathcal{N}}=W_{j \mathcal{N}}\left(\hat{\theta}_{N}\right) W_{\mathcal{N N}}^{-1}\left(\hat{\theta}_{N}\right)$

For this algorithm the following result can be formulated:

Proposition 5 Consider a dynamic network that satisfies Assumption 1. Algorithm 5 leads to a consistent estimate of $G_{j \mathcal{N}}^{0}$ if the following conditions are satisfied:

(a) Noise $v_{j}$ is present and uncorrelated to all noise signals $v_{k}, k \in \mathcal{V}_{j} \backslash\{j\}$.

(b) The process $v_{\mathcal{N}}+\tilde{G}_{\mathcal{N Z}}^{0} v_{\mathcal{Z}}$ is full rank.

(c) Every loop through node $k, k \in\{\mathcal{N}, j\}$ has a delay.

(d) The system is in the model set, i.e. there exists a $\theta^{0}$ such that $W\left(\theta^{0}\right)=W^{0}$.

(e) $W(\infty, \theta)$ is parametrized such that there exists a permutation matrix

$$
\begin{aligned}
& P=\left[\begin{array}{cc}
1 & 0 \\
0 & P_{\mathcal{N N}}
\end{array}\right], \quad \text { such that }
\end{aligned}
$$

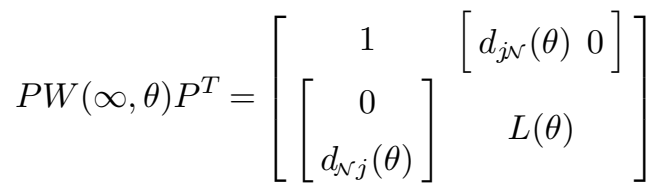

where $d_{j \mathcal{N}}(\theta)$ and $d_{\mathcal{N} j}(\theta)$ are parametrized vectors with length $\left(d_{\mathcal{N} j}\right)+\operatorname{length}\left(d_{j \mathcal{N}}\right)=\operatorname{card}\left(\mathcal{N}_{j}\right)$, and $L(\theta)$ is lower triangular with ones on the diagonal.

The result of the Proposition shows that besides the parametrization issue of Part (e), the estimation results are quite similar to the ones obtained for the direct method. Like in that method all module transfer functions $G_{j \mathcal{N}}^{0}$ need to be estimated simultaneously in order to arrive at a consistent estimate of $G_{j i}^{0}$. The delay structure conditions are tighter for the joint IO method: the set of loops that is restricted to have at least a delay is extended to all loops that run through any of the considered node signals, whereas for the direct method this only considered loops through node $j$.

The parametrization restriction formulated in Part (e) can be interpreted as follows. As mentioned before, restricting $W(\infty, \theta)$ to be $I$ is generally not leading to consistent estimates, because of algebraic off-diagonal terms that might be present in $W^{0}$. The parametrization (20) allows direct feedthrough terms to be present in the model, without the occurrence of algebraic loops that run through variables $w_{k}$, $k \in\{\mathcal{N}, j\}$. It is achieved by reordering the node signals $w_{k}, k \in \mathcal{N}$, such that the parametrized structure of (20) appears. Through the property that $\left[P W(\infty, \theta) P^{T}\right]_{k_{1} k_{2}}$. $\left[P W(\infty, \theta) P^{T}\right]_{k_{2} k_{1}}=0$ for $k_{1} \neq k_{2}$, algebraic loops in the parametrized model are avoided, while the restrictions on $W(\infty, \theta)$ are sufficient to guarantee a unique solution to the parameter estimation problem.

Whereas in the classical closed-loop situation the joint IO method is able to also estimate the feedback dynamics of the controller, in the generalized method this will typically not lead to consistent estimates of any of the module transfer functions in the network, unless $\mathcal{Z}$ is the empty set. Concerning the handling of a priori known module transfers in the estimation procedure, Remark 1 that was made concerning the direct method, applies to the Joint IO method also.

\section{Conclusions}

Several methods for closed-loop identification have been generalized to become applicable to systems that operate in a general network configuration. In the current setting we have focussed on networks in which all node signals are measurable, and where our intention is to model one particular module. Complex networks can be handled and effective use can be made of external excitation signals. These excitation signals limit the necessity to perform exhaustive consistent modelling of all noise sources in the network. The several prediction error methods presented (direct method, two-stage method based on either excitation signals or on reconstructible noise signals, and joint-IO method) are shown to be able to estimate particular subparts of the network. It opens questions as to where and how many external probing/excitation signals are required to identify particular parts of the network.

\section{Appendix}

\section{A Proof of Lemma 3.}

Let $G^{0}(\infty)$ denote $\lim _{z \rightarrow \infty} G^{0}(z)$, and let $G^{0}(\infty)$ represent a directed graph, denoted by $\mathbb{G}$. If every path from $b \rightarrow a$ has a delay then there is no path from $b$ to $a$ in the graph defined by $G^{0}(\infty)$. We can now separate the nodes of $\mathbb{G}$ into two groups, one called $\mathcal{A}$, containing node $a$ and all nodes 
that have a path to $a$, and a second group of nodes called $\mathcal{B}$, containing $b$ and all remaining nodes that have no path to $a$. By reordering the nodes in the graph $\mathbb{G}$, the matrix $G^{0}(\infty)$ related to this reordered representation can be written as

$$
G^{0}(\infty)=\left[\begin{array}{cc}
G_{\mathcal{A A}} & 0 \\
G_{\mathcal{B A}} & G_{\mathcal{B B}}
\end{array}\right]
$$

where $G_{\mathcal{A A}}$ and $G_{\mathcal{B B}}$ both have zeros on the diagonals. With the inversion rule for block matrices it follows that:

$$
(I-G(\infty))^{-1}=\left[\begin{array}{cc}
I-G_{\mathcal{A A}} & 0 \\
-G_{\mathcal{B A}} & I-G_{\mathcal{B B}}
\end{array}\right]^{-1}=\left[\begin{array}{l}
* 0 \\
* *
\end{array}\right]
$$

which shows that the $(a, b)$ entry in $\left(I-G^{0}(\infty)\right)^{-1}$ is zero. Since $\left(I-G^{0}\right)^{-1}$ is proper, this implies that the $(b, a)$ entry in $\left(I-G^{0}\right)^{-1}$ has a delay.

The reasoning to show that if there is no path from $b$ to $a$ then $\mathcal{G}_{a b}^{0}=0$ is completely analogous except that instead of working with $G^{0}(\infty)$, it is necessary to work with $G^{0}$.

\section{B Proof of Proposition 2}

The proof will proceed as follows:

(1) Show that the lower bound of the objective function $\bar{V}_{j}(\theta):=\overline{\mathbb{E}} \varepsilon_{j}^{2}(t, \theta)$ is $\sigma_{e_{j}}^{2}$, the variance of $e_{j}$.

(2) Show that $\bar{V}_{j}(\theta)=\sigma_{e_{j}}^{2}$ implies that $\theta=\theta_{0}$ (i.e the global minimum is attainable and unique).

Step 1. Throughout the proof, it will be useful to expand the measured variable $w_{i}$ in terms of all noise sources and external inputs that affect $w_{i}$. From (2b) and using the notation from Lemma 3 we have:

$$
w_{i}=\sum_{k=1}^{L} \mathcal{G}_{i k}^{0}\left(v_{k}+r_{k}\right)=\sum_{k \in \mathcal{V}_{i}} \mathcal{G}_{i k}^{0} v_{k}+\sum_{k \in \mathcal{R}_{i}} \mathcal{G}_{i k}^{0} r_{k}
$$

where the second equality holds by Lemma 3 and the definitions of $\mathcal{V}_{i}$ and $\mathcal{R}_{i}$.

Now, (B.1) will be used to express the objective function in terms of only noise sources and external inputs. With the predictor (13) it follows that

$$
\begin{aligned}
& \bar{V}_{j}(\theta)=\overline{\mathbb{E}}\left[\left(H_{j}^{-1}(\theta)\left(v_{j}+\sum_{i \in \mathcal{N}_{j}}\left(G_{j i}^{0}-G_{j i}(\theta)\right) w_{i}\right)\right)^{2}\right] \\
& =\overline{\mathbb{E}}\left[\left(H_{j}^{-1}(\theta)\left(v_{j}+\sum_{i \in \mathcal{N}_{j}} \Delta G_{j i}(\theta)\left(\sum_{k \in \mathcal{V}_{i}} \mathcal{G}_{i k}^{0} v_{k}+\sum_{k \in \mathcal{R}_{i}} \mathcal{G}_{i k}^{0} r_{k}\right)\right)\right)^{2}\right] \\
& =\overline{\mathbb{E}}\left[\left(\Delta H_{j}(\theta) v_{j}+H_{j}^{-1}(\theta) \sum_{i \in \mathcal{N}_{j}} \sum_{k \in \mathcal{V}_{i}} \Delta G_{j i}(\theta) \mathcal{G}_{i k}^{0} v_{k}\right.\right. \\
& \left.\left.\quad+H_{j}^{-1}(\theta) \sum_{i \in \mathcal{N}_{j}} \sum_{k \in \mathcal{R}_{i}} \Delta G_{j i}(\theta) \mathcal{G}_{i k}^{0} r_{k}+e_{j}\right)^{2}\right]
\end{aligned}
$$

where $\Delta G_{j i}(\theta)=G_{j i}^{0}-G_{j i}(\theta)$, and $\Delta H_{j}(\theta)=H_{j}^{-1}(\theta)-$ $H_{j}^{0^{-1}}$. Next Condition (c) will be used to simplify this expression.

By (c) if $G_{j i}^{0}$ has a delay, then $G_{j i}(\theta)$ will be parameterized with a delay (i.e. $\Delta G_{j i}(\theta)$ has a delay if $G_{j i}^{0}$ has a delay). Moreover, by Lemma 3 the term $G_{j i}^{0} \mathcal{G}_{i j}$ has a delay if all paths from $j$ to $j$ have a delay. By Condition (c), every path from $j$ to $j$ has a delay, therefore, $\Delta G_{j i}(\theta) \mathcal{G}_{i j}^{0}$ has a delay for all $i$.

Consequently every term in (B.2) is uncorrelated to $e_{j}$ :

- since $H_{j}(\theta)$ and $H_{j}^{0}$ are both monic, $\Delta H_{j}(\theta) v_{j}$ is a function of $v_{j}(t-k), k>1$;

- as described above, $\Delta G_{j i}(\theta) \mathcal{G}_{i j}^{0} v_{j}$ is also a function of $v_{j}(t-k) k>1$;

- by Condition (b) any term involving $v_{k}, k \in \mathcal{V}_{j}, k \neq j$ is uncorrelated to $e_{j}$;

- by Condition a of Assumption $1, e_{j}$ is uncorrelated to $r_{k}$ for all $k$.

Using this reasoning to simplify (B.2) results in:

$$
\begin{array}{r}
\bar{V}_{j}(\theta)=\overline{\mathbb{E}}\left[\left(\Delta H_{j}(\theta) v_{j}+H_{j}^{-1}(\theta) \sum_{i \in \mathcal{N}_{j}} \sum_{k \in \mathcal{V}_{i}} \Delta G_{j i}(\theta) \mathcal{G}_{i k}^{0} v_{k}\right.\right. \\
\left.\left.+H_{j}^{-1}(\theta) \sum_{i \in \mathcal{N}_{j}} \sum_{k \in \mathcal{R}_{i}} \Delta G_{j i}(\theta) \mathcal{G}_{i k}^{0} r_{k}\right)^{2}\right]+\sigma_{e_{j}}^{2} \\
=\overline{\mathbb{E}}\left[\left(\Delta H_{j}(\theta) v_{j}+H_{j}^{-1}(\theta) \sum_{i \in \mathcal{N}_{j}} \Delta G_{j i}(\theta) w_{i}\right)^{2}\right]+\sigma_{e_{j}}^{2}
\end{array}
$$

where $\sigma_{e_{j}}^{2}$ is the variance of $e_{j}$. From (B.3), it is clear that $\bar{V}_{j}(\theta) \geq \sigma_{e_{j}}^{2}$. This concludes the first step.

Step 2. Next it must be shown that the global minimum of $\bar{V}_{j}(\theta)$ is attainable and unique. This will be done by showing

$$
\bar{V}_{j}(\theta)=\sigma_{e_{j}}^{2} \Rightarrow \theta=\theta_{0}
$$

Using (B.3), $\bar{V}_{j}(\theta)=\sigma_{e_{j}}^{2}$ can be written as

$$
\left.\overline{\mathbb{E}}\left[\sum_{i \in \mathcal{N}_{j}} \frac{\Delta G_{j i}(\theta)}{H_{j}(\theta)} w_{i}+\Delta H_{j}(\theta) v_{j}\right)^{2}\right]+\sigma_{e_{j}}^{2}=\sigma_{e_{j}}^{2}
$$

or equivalently

$$
\overline{\mathbb{E}}\left[\left(\left[\Delta H_{j}(\theta) \frac{\Delta G_{j n_{1}}(\theta)}{H_{j}(\theta)} \cdots \frac{\Delta G_{j n_{\mathfrak{n}}}(\theta)}{H_{j}(\theta)}\right]\left[\begin{array}{c}
v_{j} \\
w_{n_{1}} \\
\vdots \\
w_{n_{\mathfrak{n}}}
\end{array}\right]\right)^{2}\right]=0
$$




$$
\begin{aligned}
& \overline{\mathbb{E}}\left[\left(\Delta x(\theta)\left[\begin{array}{ccc}
1-G_{j n_{1}}^{0} & \cdots & -G_{j n_{\mathfrak{n}}}^{0} \\
1 & & \\
& \ddots & \\
& & 1
\end{array}\right]\left[\begin{array}{c}
w_{j} \\
w_{n_{1}} \\
\vdots \\
w_{n_{\mathfrak{n}}}
\end{array}\right]\right)^{2}\right]=0 \\
& \overline{\mathbb{E}}\left[\left(\Delta x(\theta)^{T} J w_{\left\{j, \mathcal{N}_{j}\right\}}\right)^{2}\right]=0
\end{aligned}
$$

where

$$
\begin{aligned}
& \Delta x(\theta)^{T}=\left[\Delta H_{j}(\theta) \frac{\Delta G_{j n_{1}}(\theta)}{H_{j}(\theta)} \cdots \frac{\Delta G_{j n_{\mathrm{n}}}(\theta)}{H_{j}(\theta)}\right], \\
& w_{\left\{i, \mathcal{N}_{j}\right\}}^{T}=\left[\begin{array}{llll}
w_{j} & w_{n_{1}} & \cdots & w_{n_{\mathfrak{n}}}
\end{array}\right], n_{k} \in \mathcal{N}_{j} .
\end{aligned}
$$

Using Parseval's Theorem results in:

$$
\frac{1}{2 \pi} \int_{-\pi}^{\pi} \Delta x\left(e^{j \omega}, \theta\right)^{T} J \Phi_{\left\{j, \mathcal{N}_{j}\right\}}(\omega) J^{*} \Delta x\left(e^{-j \omega}, \theta\right) \mathrm{d} \omega=0
$$

for $\omega \in[-\pi, \pi)$, where $J^{*}$ denotes the conjugate transpose of $J$. By Condition (d), $\Phi_{\left\{j, \mathcal{N}_{j}\right\}}(\omega)$ is positive definite. Moreover, $J\left(e^{j \omega}\right)$ is full rank for all $\omega$. Thus the only way the equation can hold is if each entry of $\left[\begin{array}{llll}\Delta H_{j} & \Delta G_{j n_{1}} & \cdots & \Delta G_{j n_{n_{1}}}\end{array}\right]$ is equal to zero for all $\omega$. Therefore, by Condition (e) and if the parameterization of $G_{j i}(\theta)$ is such that the only way that $G_{j i}^{0}-G_{j i}(\theta)$ is equal to zero is when $G_{j i}(\theta)=G_{j i}^{0}$, the global minimum of $\bar{V}_{j}(\theta)$ is unique.

Remark A.1 There exists an alternative reasoning to prove the proposition, by utilizing the equivalent feedback structure as presented in Proposition 1, combined with the reasoning in [21] concerning absence of algebraic loops. However the proof presented above naturally includes the excitation conditions also.

\section{Proof of Lemma 4}

The variables $w_{\mathcal{z}}$ can be eliminated from the equations:

$$
\begin{aligned}
& {\left[\begin{array}{l}
w_{j} \\
w_{\mathcal{N}}
\end{array}\right]=\left[\begin{array}{cc}
0 & G_{j \mathcal{N}}^{0} \\
G_{\mathcal{N} j}^{0} & G_{\mathcal{N N}}^{0}
\end{array}\right]\left[\begin{array}{l}
w_{j} \\
w_{\mathcal{N}}
\end{array}\right]+\left[\begin{array}{c}
0 \\
G_{\mathcal{N Z}}^{0}
\end{array}\right]\left(I-G_{\mathcal{Z Z}}^{0}\right)^{-1}\left[G_{z j}^{0} G_{\mathcal{Z}}^{0}\right]\left[\begin{array}{l}
w_{j} \\
w_{\mathcal{N}}
\end{array}\right]} \\
& +\left[\begin{array}{c}
0 \\
G_{\mathcal{N Z}}^{0}
\end{array}\right]\left(I-G_{\mathcal{Z Z}}^{0}\right)^{-1} v_{\mathcal{Z}}+\left[\begin{array}{l}
v_{j} \\
v_{\mathcal{N}}
\end{array}\right] \\
& =\left[\begin{array}{cc}
0 & G_{j \mathcal{N}}^{0} \\
\tilde{G}_{\mathcal{N} j}^{0} & \tilde{G}_{\mathcal{N N}}^{0}
\end{array}\right]\left[\begin{array}{c}
w_{j} \\
w_{\mathcal{N}}
\end{array}\right]+\left[\begin{array}{ccc}
I & 0 & 0 \\
0 & I & \tilde{G}_{\mathcal{N Z}}^{0}
\end{array}\right]\left[\begin{array}{c}
v_{j} \\
v_{\mathcal{N}} \\
v_{\mathcal{Z}}
\end{array}\right],
\end{aligned}
$$

where the several matrices $\tilde{G}$ are implicitly defined through the equations. The transfer from $w_{\mathcal{N}}$ to $w_{j}$ is still $G_{j \mathcal{N}}^{0}$, whereas the transfer from $w_{j}$ to $w_{\mathcal{N}}$ has become a composite function of various tranfers (denoted $\tilde{G}_{\mathcal{N} j}^{0}$ ). Subsequently the map from $v$ to $\left[w_{j} w_{\mathcal{N}}\right]^{T}$ is

$$
\left[\begin{array}{c}
w_{j} \\
w_{\mathcal{N}}
\end{array}\right]=\left[\begin{array}{cc}
1 & -G_{j \mathcal{N}}^{0} \\
-\tilde{G}_{\mathcal{N} j}^{0} & I-\tilde{G}_{\mathcal{N N}}^{0}
\end{array}\right]^{-1}\left[\begin{array}{ccc}
I & 0 & 0 \\
0 & I & \tilde{G}_{\mathcal{N Z}}^{0}
\end{array}\right]\left[\begin{array}{l}
v_{j} \\
v_{\mathcal{N}} \\
v_{\mathcal{Z}}
\end{array}\right] .
$$

Consider the stochastic process $v_{\mathcal{N}}+\tilde{G}_{\mathcal{N Z}}^{0} v_{\mathcal{Z}}$ which appears as part of $w_{\mathcal{N}}$. Denote the power spectral density of this process as $\tilde{\Phi}_{\mathcal{N}}(\omega)$, and let $\tilde{H}_{\mathcal{N}}^{0}$ be its monic, stable and minimumphase spectral factor. Substituting this into the expression of the data generating system results in

$$
\left[\begin{array}{c}
w_{j} \\
w_{\mathcal{N}}
\end{array}\right]=\left[\begin{array}{cc}
\breve{G}_{j j}^{0} & G_{j \mathcal{N}}^{0} \breve{G}_{\mathcal{N N}}^{0} \\
\breve{G}_{\mathcal{N N}}^{0} \tilde{G}_{\mathcal{N} j}^{0} & \breve{G}_{\mathcal{N N}}^{0}
\end{array}\right]\left[\begin{array}{c}
H_{j}^{0} \\
\tilde{H}_{\mathcal{N}}^{0}
\end{array}\right]\left[\begin{array}{c}
e_{j} \\
\tilde{e}_{\mathcal{N}}
\end{array}\right]
$$

where $e_{j}$ and $\tilde{e}_{\mathcal{N}}$ are uncorrelated since $v_{j}$ and $v_{\mathcal{N}}$ and $v_{\mathcal{Z}}$ are uncorrelated.

\section{Proof of Proposition 5}

Before proceeding to the proof, consider the following useful lemmas.

Lemma A.1 Consider a dynamic network that satisfies Assumption 1. If every path from $w_{k_{2}} \rightarrow w_{k_{1}}, k_{1} \neq k_{2} \in$ $\left\{j, \mathcal{N}_{j}\right\}$ has a delay, then $\left[W^{0}\right]_{k_{1} k_{2}}$ has a delay. If every path from $w_{k} \rightarrow w_{k}, k \in\left\{j, \mathcal{N}_{j}\right\}$ has a delay then $\left[W^{0}\right]_{k k}$ is a monic transfer function.

Proof: The result follows directly from the combination of Lemmas 3 and 4.

Lemma A.2 Consider a dynamic network that satisfies Assumption 1. If every loop that runs through $k \in\{j, \mathcal{N}\}$ has a delay then there exists a permutation matrix $P$ structured as (19) such that $P W^{0}(\infty) P^{T}$ is structured according to (20).

Proof: Since we are assuming that every loop through $w_{k}$, $k \in\left\{j, \mathcal{N}_{j}\right\}$ has a delay, it follows by Lemma A.1 that the diagonal entries of $W^{0}$ are monic transfers. This proves the left upper part of (20).

Consider a graph of $W_{\mathcal{N N}}^{0}(\infty)-I$. By Lemma A.1, since every loop involving $w_{k}, k \in\left\{j, \mathcal{N}_{j}\right\}$ has a delay this is an acyclic graph, and thus (see [7]) there exists a permutation matrix $P_{\mathcal{N N}}$ such that $P_{\mathcal{N N}} W_{\mathcal{N N}}^{0}(\infty) P_{\mathcal{N N}}^{T}$ is lower triangular, conforming to the right lower part of (20). Note that $P_{\mathcal{N N}}$ may not be unique. Let $\mathcal{P}_{\mathcal{N N}}$ denote the set of permutation matrices that satisfies the above condition.

Then it must be shown that for at least one $P_{\mathcal{N N}} \in \mathcal{P}_{\mathcal{N N}}$, the off-diagonal blocks are structured according to (20). The reasoning will be split into two steps. First it will be shown 
that there exists a $P_{\mathcal{N N}} \in \mathcal{P}_{\mathcal{N N}}$ such that $P_{\mathcal{N N}} W_{\mathcal{N} j}^{0} W_{j \mathcal{N}}^{0} P_{\mathcal{N N}}^{T}$ is strictly lower triangular. Then it will be shown that for this matrix to be strictly lower triangular, the structure of (20) must hold.

Consider the graph of $W_{\mathcal{N N}}^{0}(\infty)+W_{\mathcal{N} j}^{0}(\infty) W_{j \mathcal{N}}^{0}(\infty)-I$. This graph is equal to the original graph of $W_{\mathcal{N N}}^{0}(\infty)-I$ with some new edges added. The set of permutation matrices that triangularizes the corresponding matrix, will be a subset of $\mathcal{P}_{\mathcal{N N}}$ since edges have only been added to $W_{\mathcal{N N}}^{0}(\infty)-I$ and none have been removed. This implies that if it is not triangularizable by any $P_{\mathcal{N N}} \in \mathcal{P}_{\mathcal{N N}}$, then there does not exist a permutation matrix such that it is triangularized.

Denote $P_{\mathcal{N N}}^{\prime} \in \mathcal{P}_{\mathcal{N N}}^{\prime} \subseteq \mathcal{P}_{\mathcal{N N}}$ as the set of permutation matrices that triangularize $W_{\mathcal{N} \mathcal{N}}^{0}(\infty)+W_{\mathcal{N} j}^{0}(\infty) W_{j \mathcal{N}}^{0}(\infty)-I$. By the condition that all loops passing through $w_{k}, k \in\left\{j, \mathcal{N}_{j}\right\}$ have a delay, the graph $W_{\mathcal{N N}}^{0}(\infty)+W_{\mathcal{N} j}^{0}(\infty) W_{j \mathcal{N}}^{0}(\infty)-I$ is acyclic. Then this implies ([7]) that there exists a permutation matrix $P_{\mathcal{N N}}^{\prime}$ such that

$$
P_{\mathcal{N N}}^{\prime}\left(W_{\mathcal{N N}}^{0}(\infty)+W_{\mathcal{N} j}^{0}(\infty) W_{j \mathcal{N}}^{0}(\infty)-I\right) P_{\mathcal{N N}}^{\prime T}
$$

is lower triangular. Consequently $\mathcal{P}_{\mathcal{N N}}$ is not empty. Since $\mathcal{P}_{\mathcal{N N}}^{\prime} \subseteq \mathcal{P}_{\mathcal{N N}}$ it follows that there exists a permutation matrix such that $P_{\mathcal{N N}}^{\prime} W_{\mathcal{N N}}^{0}(\infty) P_{\mathcal{N N}}^{\prime T}$ and $P_{\mathcal{N N}}^{\prime} W_{\mathcal{N} j}^{0}(\infty) W_{j \mathcal{N}}^{0}(\infty) P_{\mathcal{N N}}^{\prime T}$ are both lower triangular.

From Lemma A.1 it follows that the diagonal entries of $P_{\mathcal{N N}}^{\prime} W_{\mathcal{N} j}^{0}(\infty) W_{j \mathcal{N}}^{0}(\infty) P_{\mathcal{N N}}^{\prime T}$ are zero and therefore this matrix is strictly lower triangular. Next it will be shown that the fact that $P_{\mathcal{N N}}^{\prime} W_{\mathcal{N} j}^{0} W_{j \mathcal{N}}^{0} P^{\prime T}{ }_{\mathcal{N N}}^{T}$ is strictly lower triangular implies the off-diagonal structure of (20). Consider two vectors, $x^{T}=\left[\begin{array}{ll}x_{1}^{T} & x_{2}^{T}\end{array}\right]$ and $y^{T}=\left[\begin{array}{ll}y_{1}^{T} & y_{2}^{T}\end{array}\right]$. Then,

$$
\left[\begin{array}{l}
x_{1} \\
x_{2}
\end{array}\right]\left[\begin{array}{ll}
y_{1}^{T} & y_{2}^{T}
\end{array}\right]=\left[\begin{array}{ll}
x_{1} y_{1}^{T} & x_{1} y_{2}^{T} \\
x_{2} y_{1}^{T} & x_{2} y_{2}^{T}
\end{array}\right]
$$

The only way this matrix can be strictly lower triangular is if both $x_{1}$ and $y_{2}$ are zero. Let $x=P_{\mathcal{N N}}^{\prime} W_{\mathcal{N} j}^{0}(\infty)$ and $y=W_{j \mathcal{N}}^{0}(\infty) P_{\mathcal{N N}}^{\prime T}$, then by this reasoning, the structure of the off-diagonal blocks in (20) follows.

The proof of Proposition 5 proceeds in the usual fashion:

(1) Calculate a lower bound on $\bar{V}(\theta)$.

(2) Show that achieving this lower bound implies that $\theta=$ $\theta_{0}$.

Step 1. The expression for $\bar{V}(\theta)$ is

$$
\begin{aligned}
\bar{V}(\theta) & =\overline{\mathbb{E}}\left[\varepsilon^{T}(t, \theta) \varepsilon(t, \theta)\right]=\operatorname{tr}\left\{\overline{\mathbb{E}}\left[\varepsilon(t, \theta) \varepsilon^{T}(t, \theta)\right]\right\} \\
& =\operatorname{tr}\left\{\overline{\mathbb{E}}\left[W(\theta)^{-1} w w^{T} W(\theta)^{T}\right]\right\}
\end{aligned}
$$

with $w:=\left[\begin{array}{ll}w_{j} & w_{\mathcal{N}}^{T}\end{array}\right]^{T}$. Then with $Q:=\operatorname{cov}(e)$,

$$
\bar{V}(\theta)=\frac{1}{2 \pi} \int_{-\pi}^{\pi} \operatorname{tr}\left\{W(\theta)^{-1} W^{0} Q\left(W(\theta)^{-1} W^{0}\right)^{*}\right\} d \omega .
$$

Now consider the LDU-decomposition of the symmetric matrix $Q: Q=L_{Q} D L_{Q}^{T}$ with $L_{Q}$ lower triangular with ones on the diagonal, and $D$ diagonal, then because of the structure of $e, L_{Q}$ will be a block diagonal matrix with diagonal blocks $\left(1, L_{\mathcal{N N}}^{Q}\right)$ and $L_{\mathcal{N} \mathcal{N}}^{Q}$ lower triangular with ones on the diagonal.

The expression for $\bar{V}(\theta)$ can be expanded as:

$$
\begin{aligned}
& \bar{V}(\theta)=\frac{1}{2 \pi} \int_{-\pi}^{\pi} \operatorname{tr}\left\{\left(W(\theta)^{-1} W^{0} L_{Q}-I\right) D\left(W(\theta)^{-1} W^{0} L_{Q}-I\right)^{*}\right. \\
& \left.+\left(W(\theta)^{-1} W^{0} L_{Q}-I\right) D+D\left(W(\theta)^{-1} W^{0} L_{Q}-I\right)^{*}+D\right\} \mathrm{d} \omega
\end{aligned}
$$

Two important properties of (D.2) is that the first term is $\geq 0$ for any $\theta$, and secondly that the last term is not a function of $\theta$. In the following text it will be shown that the second and third terms of (D.2) are zero. Consequently, $\bar{V}(\theta) \geq \operatorname{tr}\{D\}$. Consider the second term of (D.2), while using the matrix inversion lemma for $W(\theta)^{-1}$ :

$$
\begin{aligned}
& \left(\begin{array}{ll}
A & B \\
C & D
\end{array}\right)^{-1}= \\
& =\left(\begin{array}{cc}
A-B D^{-1} C & 0 \\
0 & D-C A^{-1} B
\end{array}\right)^{-1}\left(\begin{array}{cc}
I & -B D^{-1} \\
-C A^{-1} & I
\end{array}\right)
\end{aligned}
$$

then $\left(W(\theta)^{-1} W^{0} L_{Q}-I\right) D=$

$$
\begin{array}{r}
\left(\left[\begin{array}{cc}
W_{j j}(\theta)-W_{j \mathcal{N}}(\theta) W_{\mathcal{N N}}^{-1}(\theta) W_{\mathcal{N} j}(\theta) & 0 \\
0 & W_{\mathcal{N N}}(\theta)-W_{\mathcal{N} j}(\theta) W_{j j}^{-1}(\theta) W_{j \mathcal{N}}(\theta)
\end{array}\right]^{-1}\right. \\
\cdot\left[\begin{array}{ll}
W_{j j}^{0}-W_{j \mathcal{N}}(\theta) W_{\mathcal{N N}}^{-1}(\theta) W_{\mathcal{N} j}^{0} & W_{j \mathcal{N}}^{0}-W_{j \mathcal{N}}(\theta) W_{\mathcal{N N}}^{-1}(\theta) W_{\mathcal{N N}}^{0} \\
W_{\mathcal{N} j}^{0}-W_{\mathcal{N} j}(\theta) W_{j j}^{-1}(\theta) W_{j j}^{0} & W_{\mathcal{N N}}^{0}-W_{\mathcal{N} j}(\theta) W_{j j}^{-1}(\theta) W_{j \mathcal{N}}^{0}
\end{array}\right] \\
\left.\cdot\left[\begin{array}{cc}
1 & 0 \\
0 & L_{\mathcal{N N}}^{\mathcal{Q}}
\end{array}\right]-I\right) D
\end{array}
$$

then the first diagonal term

$$
\begin{aligned}
\left(W_{j j}(\theta)-W_{j \mathcal{N}}(\theta)\right. & \left.W_{\mathcal{N N}}^{-1}(\theta) W_{\mathcal{N} j}(\theta)\right)^{-1} \\
& \cdot\left(W_{j j}^{0}-W_{j \mathcal{N}}(\theta) W_{\mathcal{N N}}^{-1}(\theta) W_{\mathcal{N} j}^{0}\right)-1
\end{aligned}
$$

is a strictly proper transfer function, which means that the first diagonal element of $\left(W(\theta)^{-1} W^{0} L_{Q}-I\right) D$ is a strictly proper transfer function.

Secondly,

$$
\begin{aligned}
&\left(W_{\mathcal{N N}}(\theta)-W_{\mathcal{N} j}(\theta)\right.\left.W_{j j}^{-1}(\theta) W_{j \mathcal{N}}(\theta)\right)^{-1} \\
& \cdot\left(W_{\mathcal{N N}}^{0}-W_{\mathcal{N} j}(\theta) W_{j j}^{-1}(\theta) W_{j \mathcal{N}}^{0}\right) L_{\mathcal{N N}}^{\mathcal{Q}}
\end{aligned}
$$

is a product of three lower triangular matrices with ones on the diagonals. This is induced by Condition (e), and Lemmas A.1 and A.2. The statement follows, since the inverse 
of a product of triangular matrices with ones on the diagonal, is a lower triangular matrix with ones on the diagonal. Consequently, the diagonal terms of $\left(W(\theta)^{-1} W^{0} L_{Q}-I\right) D$ are all strictly proper transfer functions.

Finally, since $\int_{-\pi}^{\pi}\left[F\left(e^{i \omega}\right)+F^{*}\left(e^{i \omega}\right)\right] d \omega=0$ for strictly proper $F$, (D.2) can be simplified to

$$
\begin{array}{r}
\bar{V}(\theta)=\frac{1}{2 \pi} \int_{-\pi}^{\pi} \operatorname{tr}\left\{\left(W(\theta)^{-1} W^{0} L_{Q}-I\right) D\left(W(\theta)^{-1} W^{0} L_{Q}-I\right)^{*}\right. \\
+D\} \mathrm{d} \omega \geq \operatorname{tr}\{D\} \quad \text { (D.3) }
\end{array}
$$

Step 2. From (D.3) $\bar{V}(\theta)=\operatorname{tr}\{D\}$ implies that

$$
\frac{1}{2 \pi} \int_{-\pi}^{\pi} \operatorname{tr}\left\{\left(W(\theta)^{-1} W^{0} L_{Q}-I\right) D\left(W(\theta)^{-1} W^{0} L_{Q}-I\right)^{*}\right\} \mathrm{d} \omega=0
$$

Since $D$ is positive definite $\forall \omega \in[-\pi, \pi)$ this implies that $\left(W(\theta)^{-1} W^{0} L_{Q}-I\right)=0 \forall \omega \in[-\pi, \pi)$. Consquently,

$$
\begin{aligned}
W(\theta) & =W^{0} L_{Q} \quad \text { for all } \omega \in[-\pi, \pi) \\
{\left[\begin{array}{ll}
W_{j j}(\theta) & W_{j \mathcal{N}}(\theta) \\
W_{\mathcal{N} j}(\theta) & W_{\mathcal{N N}}(\theta)
\end{array}\right] } & =\left[\begin{array}{ll}
W_{j j}^{0} & W_{j \mathcal{N}}^{0} L_{\mathcal{N N}}^{\mathcal{Q}} \\
W_{\mathcal{N} j}^{0} & W_{\mathcal{N N}}^{0} L_{\mathcal{N N}}^{\mathcal{Q}}
\end{array}\right]
\end{aligned}
$$

By Condition (e) and Lemma A.2 the parameterization is such that there exists a solution to this equation. In particular the parameterization is such that the equality $W_{j \mathcal{N}}(\theta)=$ $W_{j \mathcal{N}}^{0} L_{\mathcal{N N}}^{\mathcal{Q}}$ can hold. This completes the proof.

\section{References}

[1] B. D. O. Anderson and M. R. Gevers. Identifiability of linear stochastic-systems operating under linear feedback. Automatica, 18(2):195-213, 1982.

[2] M. Araki and M. Saeki. Quantitative conditions for the wellposedness of interconnected dynamic systems. IEEE Trans. Automatic Control, 28(5):625-637, 1983.

[3] J. Gonçalves and S. Warnick. Necessary and sufficient conditions for dynamical structure reconstruction of LTI networks. IEEE Trans. Automatic Control, 53(7):1670-1674, August 2008.

[4] A. Dankers, P.M.J. Van den Hof, P.S.C. Heuberger, and X. Bombois. Dynamic network identification using the direct prediction error method. In Proc. 51th IEEE Conf. Decision and Control, pages 901-906, Maui, HI, USA, 10-13 December 2012.

[5] A. Dankers, P.M.J. Van den Hof, P.S.C. Heuberger, and X. Bombois. Dynamic network structure identification with prediction error methods - basic examples. In Proc. 16th IFAC Symposium on System Identification, pages 876-881, Brussels, Belgium, 11-13 July 2012.

[6] A. G. Dankers, P. M. J. Van den Hof, X. Bombois, and P. S. C. Heuberger. Identification of dynamic models in complex networks with prediction error methods - input selection in two-stage methods. In Proc. 2013 European Control Conference, Zürich, Switzerland, 17-19 July 2013. To appear.

[7] N. Deo. Graph Theory with Applications to Engineering and Computer Science. Prentice Hall of India, Connaught Circus, New Delhi, 1974.

[8] R. Diestel. Graph Theory. Springer Verlag, Berlin, 2006.
[9] U. Forssell and L. Ljung. Closed-loop identification revisited. Automatica, 35(7):1215-1241, 1999.

[10] U. Forssell and L. Ljung. A projection method for closed-loop identification. IEEE Trans. Automatic Control, 45(11):2101-2106, 2000.

[11] J. Friedman, T. Hastie, and R. Tibshirani. Applications of the lasso and grouped lasso to the estimation of sparse graphical models. unpublished, 2010.

[12] M. Gevers, A. Bazanella, X. Bombois, and L. Misković. Identification and the information matrix: how to get just sufficiently rich. IEEE Trans. Automatic Control, 54(12):2828-2840, 2009.

[13] M. Gilson and P. M. J. Van den Hof. Instrumental variable methods for closed-loop system identification. Automatica, 41(2):241-249, 2005.

[14] M. Leskens and P. M. J. Van den Hof. Closed-loop identification of multivariable processes with part of the inputs controlled. Int. J. Control, 80(10):1552-1561, 2007.

[15] L. Ljung. System Identification: Theory for the User. Prentice-Hall, Englewood Cliffs, NJ, 1999.

[16] D. Materassi and G. Innocenti. Topological identification in networks of dynamical systems. IEEE Trans. Automatic Control, 55(8):1860 $1871,2010$.

[17] D. Materassi, M.V. Salapaka, and L Giarrè. Relations between structure and estimators in networks of dynamical systems. In Proc. 50th IEEE Conf. Decision and Control, pages 162-167, Orlando, USA, 2011.

[18] T.S. Ng, G.C. Goodwin, and B.D.O. Anderson. Identifiability of MIMO linear dynamic systems operating in closed loop. Automatica, 13:477-485.

[19] B.M. Sanandaji, T.L. Vincent, and M.B. Wakin. Exact topology identification of large-scale interconnected dynamical systems from compressive observations. In Proceedings of American Control Conference, pages 649-656, San Francisco, CA, USA, 2011.

[20] P. M. J. Van den Hof. Closed-loop issues in system identification. Annual Reviews in Control, 22:173-186, 1998.

[21] P. M. J. Van den Hof, D. K. de Vries, and P. Schoen. Delay structure conditions for identifiability of closed loop systems. Automatica, 28(5):1047-1050, 1992.

[22] P. M. J. Van den Hof and R. J. P. Schrama. An indirect method for transfer function estimation from closed loop data. Automatica, 29(6):1523-1527, 1993.

[23] P.M.J. Van den Hof, A. Dankers, P.S.C. Heuberger, and X. Bombois. Identification in dynamic networks with known interconnection topology. In Proc. 51th IEEE Conf. Decision and Control, pages 895-900, Maui, HI, USA, 10-13 December 2012.

[24] E. Yeung, J. Gonçalves, H. Sandberg, and S. Warnick. Representing structure in linear interconnected dynamical systems. In Proc. 49th IEEE Conf. Decision and Control, pages 6010-6015, Atlanta, GA, USA, 15-17 December 2010.

[25] Y. Yuan, G.-B. Stan, S. Warnick, and J. Gonçalves. Minimal dynamical structure realisations with apllication to network reconstruction from data. In Proc. 48th IEEE Conf. Decision and Control, pages 4808-4813, Shanghai, China, 2009.

[26] Y. Yuan, G.-B. Stan, S. Warnick, and J. Gonçalves. Robust dynamical network reconstruction. In Proc. 49th IEEE Conf. Decision and Control, pages 810-815, Atlanta, USA, 2010. 